\title{
Acoustic Inversion for Uncertainty Reduction in Reynolds-Averaged Navier-Stokes-Based Jet Noise Prediction
}

\author{
Xin-Lei Zhang*๑ \\ State Key Laboratory of Nonlinear Mechanics, Chinese- Academy of Sciences, 100190 Beijing, \\ People's Republic of China \\ Heng Xiao \\ Virginia Tech, Blacksburg, Virginia 24060 \\ and \\ Ting $\mathrm{Wu}^{\sharp}$ and Guowei $\mathrm{He}^{\underline{\S}}$ \\ State Key Laboratory of Nonlinear Mechanics, Chinese Academy of Sciences, 100190 Beijing, \\ People's Republic of China \\ https://doi.org/10.2514/1.J060876
}

\begin{abstract}
The Reynolds-averaged Navier-Stokes (RANS)-based method is a practical tool to provide rapid assessment of jet noise-reduction concepts. However, the RANS-based method requires modeling assumptions to represent noise generation and propagation, which often reduces the predictive accuracy due to the model-form uncertainties. In this work, the ensemble Kalman filter-based acoustic inversion method is introduced to reduce uncertainties in the turbulent kinetic energy and dissipation rate based on the far-field noise and the axial centerline velocity data. The results show that jet noise data are more effective from which to infer turbulent kinetic energy and dissipation rate compared to velocity data. Moreover, the inferred noise source is able to improve the estimation of the turbulent flowfield and the far-field noise at unobserved locations. Further, the noise model parameters are also considered uncertain quantities, demonstrating the ability of the proposed framework to reduce uncertainties in both the RANS and noise models. Finally, one realistic case with experimental data is investigated to show the practicality of the proposed framework. The method opens up the possibility for the inverse modeling of jet noise sources by incorporating far-field noise data that are relatively straightforward to be measured compared to the velocity field.
\end{abstract}

\begin{tabular}{ll} 
& \multicolumn{1}{c}{ Nomenclature } \\
$A, c_{l}$, and $c_{\tau}$ & $=$ acoustic model coefficients \\
$c_{0}$ & $=$ ambient sound speed \\
$D$ & $=$ diameter of nozzle exit \\
$\mathcal{F}$ & $=$ acoustic model operator \\
$f$ & $=$ frequency \\
$\mathrm{H}$ & $=$ tangent linear operator \\
$\mathcal{H}$ & $=$ composite model operator \\
$\mathrm{I}$ & $=$ noise power \\
$\mathrm{K}$ & $=$ Kalman gain matrix \\
$\mathcal{K}$ & $=$ kernel function \\
$k$ & $=$ turbulent kinetic energy \\
$l$ & $=$ correlation length in the Gaussian kernel \\
$l_{s}$ & $=$ function \\
$M$ & $=$ size of ensemble \\
$\mathcal{M}^{*}$ & $=$ the Reynolds-averaged Navier-Stokes model \\
$N$ & $=$ number of modes
\end{tabular}

Received 4 May 2021; revision received 25 September 2021; accepted for publication 22 October 2021; published online 13 December 2021. Copyright (C) 2021 by the American Institute of Aeronautics and Astronautics, Inc. All rights reserved. All requests for copying and permission to reprint should be submitted to CCC at www.copyright.com; employ the eISSN 1533-385X to initiate your request. See also AIAA Rights and Permissions www.aiaa.org/ randp.

*Postdoctoral Research Associate, Institute of Mechanics; also School of Engineering Sciences, University of Chinese Academy of Sciences, 100049 Beijing, People's Republic of China.

${ }^{\dagger}$ Associate Professor, Kevin T. Crofton Department of Aerospace and Ocean Engineering.

${ }^{\ddagger}$ Postdoctoral Research Associate, Institute of Mechanics; also School of Engineering Sciences, University of Chinese Academy of Sciences, 100049 Beijing, People's Republic of China.

\$Professor, Institute of Mechanics; also School of Engineering Sciences, University of Chinese Academy of Sciences, 100049 Beijing, People's Republic of China; hgw@lnm.imech.ac.cn (Corresponding author).
$\mathrm{P}$

$p$

$q_{s}$

$\hat{q}$

$\mathrm{R}$

S

$t$

$U$
$u$

$u$
$w$

$\mathrm{X}$

$x$

$\mathrm{y}$

$\gamma$

$\delta$

$\varepsilon$

$\eta$

$\theta$

$\lambda$

$\nu_{t}$

$\rho$

$\sigma$

$\tau_{s}$

$\Phi$

$\omega$

Subscripts

$a$

n

0

Superscripts

a
$=$ covariance of model error

$=$ pressure

$=$ the unsteady pressure due to the fine-scale turbulence

$=$ the magnitudes of the turbulent fluctuation

$=$ covariance of observation error

$=$ distance between the observed position and the source field

$=$ noise spectral density

$=$ time

$=$ mean velocity

$=$ velocity

$=$ mode coefficients

$=$ state vector

$=$ spatial coordinate

$=$ observation vector

$=$ specific heat ratio

$=$ uncertainties

$=$ dissipation rate

$=$ observation noise

$=$ observation angle

$=$ eigenvalue of kernel function

$=$ eddy viscosity

$=$ density

$=$ variance

$=$ characteristic time scale

$=$ basis of Karhunen-Loève expansion

$=$ radian frequency

$=$ adjoint Green function

$=$ index of mode

$=$ baseline

$=$ analysis

$=$ forecast 
$\mathrm{T} \quad=$ transpose

= mean

\section{Introduction}

$\mathbf{J}$ ET EXHAUST noise has emerged as one of the main impediments to the further growth of air traffic, and noise reduction becomes increasingly demanding for design of modern jet engines. Numerical approaches play an important role to aid the assessment of noise-reduction concepts [1]. Over the past decades, large-eddy simulation (LES) has gained increasing popularity for jet noise prediction $[2,3]$ and has made significant contributions to the understanding of noise generation in jet flows [4,5]. However, noisereduction design with LES is still a challenge due to the high computational costs. As such, computationally efficient approaches, for example, the Reynolds-averaged Navier-Stokes (RANS)-based method [6-8], are an alternative and practical tool for design and optimization of jet noise-suppression devices.

Jet mixing noise consists of two different noise sources from finescale and large-scale turbulence [9]. The contribution from fine-scale turbulence is usually dominant for subsonic jet flows and the upstream of supersonic jet flows [10]. Various RANS-based statistical approaches (e.g., [11-15]) have been proposed to predict the jet mixing noise from the fine-scale turbulence. These methods mainly use the mean flowfields from RANS simulations to model the fluctuation magnitudes and characteristic scales of turbulence. Further, the acoustic propagation is computed to estimate the far-field noise by using Lighthill's acoustic analogy or a linear Euler equation [16]. However, these methods often make inaccurate predictions, particularly at the low- and high-frequency ranges $[17,18]$. That is likely caused by the various, often rather restrictive, assumptions in the modeling, for example, Boussinesq assumption and equilibrium between turbulence production and dissipation in the commonly used RANS models and the Lighthill's acoustic analogy and the parallel mean flow approximation in acoustic models. In addition, the RANS-based method requires modeling the space-time correlation [19] or energy spectrum [20] of the noise sources, which is usually separated into space and time variables [11] and prescribed with specific decay functions (e.g., Gaussian or exponential functions) [12]. For these reasons, it is of significant importance to reduce the model uncertainties and thus improve the predictive capability of the RANS-based methods.

In the past few years, data assimilation methods have demonstrated the success of reducing uncertainties in computational models by incorporating observation data. It is achieved through inferring uncertain terms in numerical simulations such as model corrections [21] or initial conditions [22] based on available observation. This approach has been used for improving the estimation of jet flowfields. For instance, He et al. [23] integrated two-dimensional (2-D) particle image velocimetry (PIV) measurements with RANS models to reconstruct the jet flowfields through inferring latent source terms in the conventional RANS model. Labahn et al. [24] assimilated tomographic PIV measurements into the LES to generate high-resolution full-field data. Pickering et al. [25] combined the resolvent analysis and the high-fidelity data from $\overline{\mathrm{LES}}$ to infer the optimal eddy viscosity that improves the resolvent-based model for the wave packets. Nevertheless, these works mentioned mainly use velocity measurements to reconstruct the entire flowfield or identify the linear response modes of jet flows. Compared to the velocity field, the far-field noise is relatively straightforward to measure by using microphone arrays, making it more practical to use the noise measurements for data-driven jet noise modeling. Moreover, these works focus on the reconstruction of jet noise sources, that is, turbulent flowfields, and the noise propagation is not considered. It is assumed that the jet mixing noise from fine-scale turbulence is generated from the turbulent fluctuations in jet flows [11]. Hence, it has the potential to improve the estimation of the turbulent kinetic energy (TKE) field through assimilating far-field noise measurements. To this end, in this work we investigate an acoustic inversion problem, that is, inferring turbulent kinetic energy and dissipation rate from jet noise data. Specifically, the forward problem is defined to estimate the far-field noise, for example, sound pressure level (SPL) at one location $x$, propagated from the noise sources, for example, turbulent kinetic energy and dissipation rate. Accordingly, the acoustic inversion is to solve an inverse problem where the noise sources are inferred based on the far-field noise measurements. Further, the jet flowfield and the noise at unobserved locations can be reconstructed with the inferred noise sources. The schematic of the acoustic inversion is shown in Fig. 1.

Different inference frameworks, such as adjoint-based methods and ensemble-based methods, can be used to solve inverse problems. The adjoint-based method is a commonly used optimization method and has been employed for noise-reduction design [26-29]. However, the method requires deriving and solving adjoint equations to estimate the gradient of objective functions, which leads to laborious efforts for code development and maintenance, particularly in complex computational fluid dynamics (CFD) codes. To this end, the ensemble-based method has gained popularity due to its nonintrusiveness and ease of implementation. The ensemble Kalman filter (EnKF) is one of the widely used ensemble-based methods, which adopts ensemble realizations to estimate the optimal gradient-descent direction. This method has been used for inverse modeling of different physical systems, such as viscous flow reconstruction [22], groundwater flow estimation [30], tsunami flow inference [31], and vortex modeling of separated flows [32]. In the past few years, it is also increasingly used to quantify and reduce the RANS model-form uncertainties in empirical parameters [33], eddy viscosity [34], and Reynolds stresses [35,36]. However, in this work we investigate the feasibility of EnKF for solving the acoustic inversion problem, that is, reducing the uncertainties in the RANS model and noise model.

Inferring the noise source information from noise measurements is common in the acoustic community. For instance, the acoustic noise data have been used for localization of the noise source $[37,38]$, detection of the underwater environment $[39,40]$, and inversion of wall pressure $[41,42]$. Besides, inferring optimal parameters in a noise model $[43,44]$ from noise data was also investigated. These works mainly focus on the noise propagation, while the noise generation, that is, estimation of turbulence fields, is not involved. In the present work, the acoustic inversion problem involves both noise generation and propagation by coupling the RANS method and statistical acoustic model. Specifically, the RANS method provides the characteristics of jet noise sources based on the modeled turbulent kinetic energy $k$ and dissipation rate $\varepsilon$, and, further, the noise model computes the noise propagation to estimate the far-field noise. The ensemble Kalman filter is leveraged to infer the optimal $k$ and $\varepsilon$ within the jet flows by assimilating the data of the far-field noise and the axial centerline velocity. Note that in this work, the inferred quantities, particularly the dissipation rate $\varepsilon$, are the modeled physical variables used to estimate the turbulent length and time scale of the jet noise sources. The dissipation rate $\varepsilon$ can be considered as surrogate quantities and used for jet noise modeling. In that sense, one can also infer the specific dissipation rate $\omega$ or length scale $l$ by using other turbulence models. The main contribution of the present work is to demonstrate the feasibility of using noise measurements, which are relatively straightforward to be measured, to reduce the uncertainties in the RANS-based jet noise prediction. Moreover, the EnKF-based acoustic inversion method is able to assimilate different

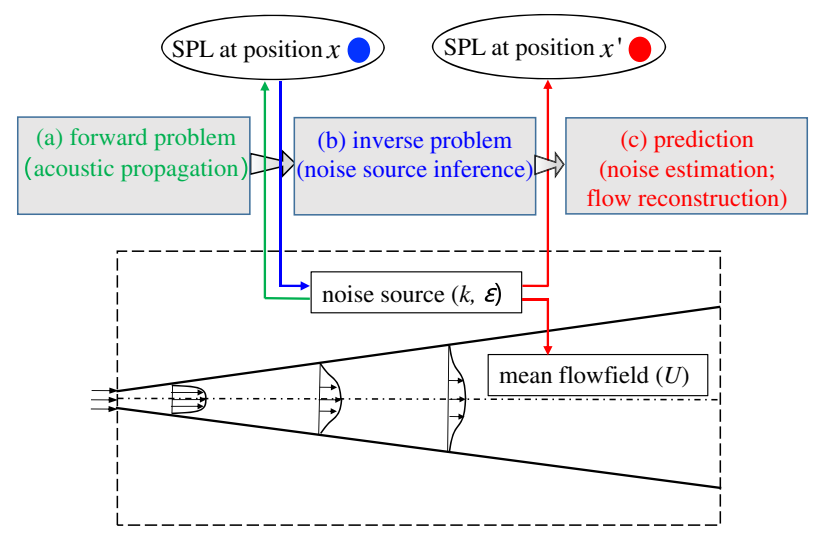

Fig. 1 Schematic of the acoustic inversion. 
data sources, including the noise and the velocity data, nonintrusively. The proposed framework opens up the possibility of reducing uncertainties in the turbulence and noise model from the noise measurements and thus significantly enriches the dataset for data-driven turbulence modeling.

The rest of this paper is structured as follows. In Sec. II, the forward problem for jet noise prediction and the EnKF-based acoustic inversion framework are described. In Sec. III, we provide the computational setup of numerical examples with different observations and inferred quantities. Numerical results are presented in Sec. IV to demonstrate the merits of the proposed framework. The computational cost, ill-posedness issue, and limitation of the present work are further discussed in Sec. V. Finally, we conclude the paper in Sec. VI.

\section{Methodology}

The goal of this work is to infer the turbulent kinetic energy and dissipation rate of the jet flow from the far-field noise, which is an inverse problem. To solve the inverse problem, the EnKF method requires solving the forward problem (e.g., the RANS-based jet noise prediction herein) a number of times to estimate the gradient-descent direction for optimal solutions. In this section, we describe the forward problem and the inverse problem of jet noise prediction and corresponding solution algorithms.

\section{A. Formulation of the Forward Problem and Solution Algorithm}

In the forward problem, the RANS equations are first solved to obtain the mean flowfields of velocity $U$, density $\bar{\rho}$, and the turbulent kinetic energy $k$ and dissipation rate $\varepsilon$. Further, the sound propagation is computed analytically to estimate the far-field noise with semiempirical noise models. We choose the conventional $k-\varepsilon$ model [45] to close the RANS equations and Tam and Auriault's model [11] to predict the far-field noise. Tam and Auriault's model assumes that the pressure fluctuation generated by the fine-scale turbulence is related to the kinetic energy. The noise radiation is estimated by solving linearized Euler equations as

$$
\bar{\rho}\left[\frac{\partial u_{i}}{\partial t}+U_{j} \frac{\partial u_{i}}{\partial x_{j}}+u_{j} \frac{\partial U_{i}}{\partial x_{j}}\right]+\frac{\partial p}{\partial x_{i}}=-\frac{\partial q_{s}}{\partial x_{j}}
$$

where $t$ is the time, $\boldsymbol{x}$ is the spatial coordinates, $\bar{\rho}$ is the mean density of jet flow, $q_{s}=(2 / 3) \bar{\rho} k_{s}$ is the unsteady pressure due to the finescale turbulence, $k_{s}$ is the kinetic energy of the fine-scale turbulence per unit mass, $U$ is the mean velocity of the jet, and $u$ and $p$ are the acoustic field variables associated with $k_{s}$. Further, the linearized equation of energy and continuity and the equation of state for a perfect gas can be written as

$$
\frac{\partial p}{\partial t}+U_{j} \frac{\partial p}{\partial x_{j}}+\gamma \bar{p} \frac{\partial u_{i}}{\partial x_{i}}=0
$$

The solution to Eqs. (1) and (2) can be found by using the space-time Green's function of these equations. Tam and Auriault [46] further use the adjoint Green's equation to describe the mean flow/acoustic interaction effects through interchanging the source and observation points. By doing this, the computational efforts can be significantly reduced, since the Green's functions for all the source points in the jet flow radiating to a given direction can be obtained in a single calculation. With the adjoint Green's function, the spectral density of the radiated sound at the observer location $\boldsymbol{x}$ can be formulated as

$$
\begin{aligned}
S(\boldsymbol{x}, \omega)= & \iint \cdots \iint_{-\infty}^{\infty} p_{a}\left(\boldsymbol{x}_{1}, \boldsymbol{x}, \omega_{1}\right) p_{a}\left(\boldsymbol{x}_{2}, \boldsymbol{x}, \omega_{2}\right) \\
& \times\left\langle\frac{\mathrm{D} q_{s}\left(\boldsymbol{x}_{1}, t_{1}\right)}{\mathrm{D} t_{1}} \frac{\mathrm{D} q_{s}\left(\boldsymbol{x}_{2}, t_{2}\right)}{\mathrm{D} t_{2}}\right\rangle \exp \left[-i\left(\omega_{1}+\omega_{2}\right) t+i \omega_{1} t_{1}\right. \\
& \left.+i \omega_{2} t_{2}\right] \delta\left(\omega-\omega_{2}\right) \mathrm{d} \omega_{1} \mathrm{~d} \omega_{2} \mathrm{~d} t_{1} \mathrm{~d} t_{2} \mathrm{~d} \boldsymbol{x}_{1} \mathrm{~d} \boldsymbol{x}_{2}
\end{aligned}
$$

where $\boldsymbol{x}_{1}$ and $\boldsymbol{x}_{2}$ represent the two locations in the noise source region, $p_{a}$ is the pressure component of the adjoint Green's function, and $\omega$ is the radian frequency. The space-time correlation

$$
\left\langle\frac{\mathrm{D} q_{s}\left(\boldsymbol{x}_{1}, t_{1}\right)}{\mathrm{D} t_{1}} \frac{\mathrm{D} q_{s}\left(\boldsymbol{x}_{2}, t_{2}\right)}{\mathrm{D} t_{2}}\right\rangle
$$

is modeled with an exponential decay in time and a Gaussian decay in space. Moreover, when the observation angle $\theta=90 \mathrm{deg}$, the effect of the mean flow is negligible, and considering the uniform sound speed, the adjoint pressure $p_{a}$ can be estimated analytically based on the Helmholtz equation [12]. Consequently, at the observation angle $\theta=90 \mathrm{deg}$, the noise spectral density is written as

$$
S(\boldsymbol{x}, \omega)=\frac{\sqrt{\pi}}{16 \pi^{2} c_{0}^{4} r^{2}} \iiint \frac{\hat{q}_{s}^{2}}{c^{2} \tau_{s}} \ell_{s}^{3} \omega^{2} \frac{\exp \left(-\omega^{2} \ell_{s}^{2} /\left(4 U_{1}^{2}\right)\right)}{\left(1+\omega^{2} \tau_{s}^{2}\right)} \mathrm{d} \boldsymbol{x}_{2}
$$

where $c_{0}$ is ambient sound speed, $r$ is the distance between the observed position and the source field, and the compact approximation $r=\left|\boldsymbol{x}-\boldsymbol{x}_{2}\right| \approx|\boldsymbol{x}|$ is applied. In the preceding formula, the magnitudes of turbulent fluctuation $\hat{q}_{s}^{2} / c^{2}$ and the characteristic length scale $l_{s}$ and time scale $\tau_{s}$ are assumed as

$$
\frac{\hat{q}_{s}^{2}}{c^{2}}=\frac{4}{9} A^{2} \bar{\rho}^{2} k^{2}, \quad l_{s}=c_{l} \frac{k^{3 / 2}}{\varepsilon}, \quad \tau_{s}=c_{\tau} \frac{k}{\varepsilon}
$$

where $A, c_{l}, c_{\tau}$ are empirical parameters and usually calibrated based on experimental measurements and $k$ and $\varepsilon$ are the turbulent kinetic energy and dissipation rate, respectively. Finally, the sound pressure level (SPL) can be estimated as

$$
\operatorname{SPL}(\boldsymbol{x}, \omega)=10 \log _{10}\left(\frac{S(\boldsymbol{x}, \omega)}{p_{\mathrm{ref}}^{2}}\right)
$$

where $p_{\text {ref }}$ is the reference pressure of $2 \times 10^{-5} \mathrm{~Pa}$ in the air. Also, we define the noise power $I$ as the integration of noise spectral density for a given frequency bandwidth $\left[f_{1}, f_{2}\right]$ as

$$
\mathbf{I}=\int_{f_{1}}^{f_{2}} \operatorname{SPL} \mathrm{d} f
$$

where the frequency $f=\omega / 2 \pi$. Note that the noise power is typically defined as the integral of noise spectral density over the given frequency bandwidth. Here, we define the specific noise power as Eq. (7), which is to investigate the effectiveness of assimilating the integral of the SPL spectra, in contrast to assimilating the SPL spectra.

In summary, we need to perform two steps to solve the forward problem of jet noise prediction. First, the RANS equation is solved to obtain the mean flowfields of velocity $U_{1}$ and density $\bar{\rho}$ given the TKE $k$ and dissipation rate $\varepsilon$ without involving the turbulence model. Specifically, this step can be formulated as

$$
z=\mathcal{M}^{*}[\mathrm{q}]
$$

where $\mathbf{q}=\{k, \varepsilon\}$ is the model inputs, $\mathbf{z}=\left\{U_{1}, \bar{\rho}\right\}$ is the model outputs, and $\mathcal{M}^{*}$ represents the RANS model operator that maps the $k$ and $\varepsilon$ onto the velocity $U_{1}$ and density $\bar{\rho}$. Further, the modeled $k$ and $\varepsilon$ are used to estimate the magnitudes of turbulent fluctuation $\hat{q}_{s}^{2} / c^{2}$, the characteristic length scale $l_{s}$, and the time scale $\tau_{s}$, given the acoustic model parameters $A, c_{l}, c_{\tau}$. The mean flowfields and the estimated noise sources are used to compute the noise spectra as

$$
\mathrm{SPL}=\mathcal{F}[\mathrm{q}, \mathrm{z}]
$$

where $\mathcal{F}$ is the model operator mapping the CFD outputs onto the farfield noise spectra. Note that the operator $\mathcal{F}$ also depends on the empirical acoustic model parameters.

\section{B. Formulation of the Inverse Problem and Solution Algorithm}

In the formulation of the forward problem, the RANS-modeled TKE $k$ and dissipation rate $\varepsilon$ often lead to large discrepancies in the prediction of jet mixing noise. To this end, we consider the uncertainty in the $k$ and $\varepsilon$ from RANS simulations. The inverse problem 
can be recast as inferring the TKE $k$ and dissipation rate $\varepsilon$ from the observation data of far-field noise and axial centerline velocity. We use the ensemble Kalman method to infer the fields of $k$ and $\varepsilon$ by incorporating observation data.

The challenge of this acoustic inversion is due to the high dimension. Unlike the typical acoustic inversion problems such as inferring locations of noise source [37] or identifying noise model parameters [44], where the inferred quantities are in a low-dimensional space, the problem in this work is to infer the turbulence fields within the noise emission region, which is in a high-dimensional space. The high dimensionality causes the ill-posedness of the field inversion. That is, the solution of the inverse problem is not unique, and different turbulence fields may lead to similar noise spectra. On the other hand, the inferred quantities, for example, TKE, are in the physical space, while the noise measurements are the integral data source in the spectral space. Inferring the physical fields with the integral-type observation may further increase the ill-posedness of the inverse problem. Additionally, the RANS-based approach couples the RANS equation and the acoustic model, which increases the nonlinearity of the system and thus the difficulty of the inversion.

To solve this inverse problem, we first consider the uncertainty in the turbulent kinetic energy $k$ and dissipation rate $\varepsilon$ as

$$
\log k=\log k_{0}+\delta_{k} \quad \log \varepsilon=\log \varepsilon_{0}+\delta_{\varepsilon}
$$

where $k_{0}$ and $\varepsilon_{0}$ indicate the initial TKE and dissipation rate, and $\delta$ represents the uncertainty. Both the $\delta_{k}$ and $\delta_{\varepsilon}$ are considered to conform to the Gaussian process $\mathcal{G P}(0, \mathcal{K})$ where $\mathcal{K}$ is the kernel function, representing the spatial covariance. To represent the covariance of two different spatial locations $\boldsymbol{x}$ and $\boldsymbol{x}^{\prime}$, we employ the square exponential kernel as

$$
\mathcal{K}\left(\boldsymbol{x}, \boldsymbol{x}^{\prime}\right)=\sigma^{2} \exp \left(-\frac{\left\|\boldsymbol{x}-\boldsymbol{x}^{\prime}\right\|^{2}}{l^{2}}\right)
$$

where $\sigma$ is the variance and $l$ is the characteristic length. Further the Karhunen-Loève (KL) expansion [47] is used to represent the Gaussian process with a linear combination of orthogonal functions (i.e., KL modes). Specifically, the KL modes are constructed based on $\Phi=\sqrt{\lambda} \hat{\Phi}$, where $\lambda$ and $\hat{\Phi}$ are the eigenvalue and eigenvector of the kernel function $\mathcal{K}$, respectively. We truncate the KL modes to cover $99 \%$ of variance and accordingly use the first $N$ modes to represent the inferred fields, thereby reducing the number of inferred parameters. Thus, the $\delta$ in Eq. (10) is constructed as

$$
\delta=\sum_{n=1}^{N} \Phi_{n} w_{n}
$$

where the KL coefficients $w_{n}$ conform to the normal distribution $\mathcal{N}(0,1)$. By doing this, we can reduce the dimension of inferred quantities and simultaneously ensure the smoothness of the inferred fields, since the inferred fields are within the subspace spanned by these KL modes. In this work, both $k$ and $\varepsilon$ use the same kernel function with the constant variance and length scale to show the feasibility of the proposed method when limited prior information is used. However, it is noted that additional prior knowledge, such as large discrepancies that exist in the shear layer region, can be straightforwardly taken into account by using a predefined variance field $\sigma(x)$. Also, the uncertainties in the acoustic model parameters of $A, c_{l}, c_{\tau}$ are able to be involved in the framework by augmenting the state, that is, $\mathbf{x}=\left[\boldsymbol{w}^{k}, \boldsymbol{w}^{\varepsilon}, A, c_{l}, c_{\tau}\right]$. Afterward, the ensemble Kalman method [30] is applied to obtain the optimal state $\mathbf{x}$. The method is a variant of the standard ensemble Kalman method for solving inverse problems. The inversion problem is recast as artificial dynamics problems and solved iteratively based on the update scheme of the ensemble Kalman filter. The steps can be divided as follows:

1) For sampling of prior distribution, we generate the KL modes $\boldsymbol{\Phi}$ based on the KL expansion and further draw random samples of the KL coefficients $\boldsymbol{w}$ from normal distributions. With the combination of $\boldsymbol{\Phi}$ and $\boldsymbol{w}$, we generate the initial samples of state $\mathbf{X}=\left\{\mathbf{x}_{j}\right\}_{j=1}^{M}$ where $M$ is the number of samples. With these samples, we can estimate the error covariance $P$ at the current iteration based on

$$
\mathrm{P}=\frac{1}{M-1}(\mathrm{X}-\overline{\mathrm{X}})(\mathrm{X}-\overline{\mathrm{X}})^{\top}
$$

where $\bar{X}$ is the sample mean.

2) For the propagation, two steps are required to solve the forward problem for each realization of state $x_{j}$ as presented in Sec. II.A. First, the RANS equations are solved to obtain the mean velocity $U$ and density $\bar{\rho}$, given $k$ and $\varepsilon$ as

$$
\mathrm{z}=\mathcal{M}[\mathrm{x}]
$$

where $\mathbf{Z}=\left[U_{1}, \bar{\rho}\right], \mathbf{x}=\left[\boldsymbol{w}^{k}, \boldsymbol{w}^{\varepsilon}\right]$, and $\mathcal{M}$ represents the model operator mapping the state $\mathbf{X}$ onto the space that $\mathbf{Z}$ is in. Note that in the case of considering the uncertainties in acoustic model parameters, the empirical parameters can be embedded in the state as $\mathbf{x}=\left[\boldsymbol{w}^{k}, \boldsymbol{w}^{\varepsilon}, A, c_{l}, c_{\tau}\right]$. Afterward, the resolved mean flowfields, i.e., the velocity $U_{1}$, density $\bar{\rho}$, turbulent kinetic energy $k$, and dissipation rate $\varepsilon$, are used to estimate the far-field noise based on the statistical noise model. That is,

$$
\mathrm{SPL}=\mathcal{F}[\mathrm{x}, \mathrm{z}]
$$

We combine the two steps with the composite function SPL $=\mathcal{H}[\mathrm{x}]$ for brevity, where the operator $\mathcal{H}$ maps the state $\mathrm{x}$ onto the far-field noise spectra. Further, we reformulate the forward model to be $\mathrm{SPL}=\mathrm{Hx}$ with the tangent linear operator $\mathrm{H}$. Thus, for each realization $\mathrm{x}_{\mathrm{j}}$, we can obtain the model prediction $\mathrm{Hx}_{j}$ in the velocity and SPL based on Eqs. (14) and (15).

3) For the analysis step, the EnKF uses the model outputs $\mathrm{Hx}$ and observation data $\mathrm{y}$ to correct the state $\mathrm{x}$ with an explicit update scheme. For each sample, the state is updated based on

$$
\mathrm{x}_{j}^{a}=\mathrm{x}_{j}^{f}+\mathrm{K}\left(\mathrm{y}_{j}-\mathrm{Hx}_{j}^{f}\right)
$$

where the superscripts $a$ and $f$ indicate the analysis and forecast respectively; $\mathrm{y}_{j}$ is the observation data with random observation noises, that is, $\mathrm{y}_{j}=\mathrm{y}+\eta_{j}$ and $\eta \sim \mathcal{N}(0, \mathrm{R})$; and the Kalman gain matrix $\mathrm{K}$ is computed with the model error covariance $\mathrm{P}$ and observation error covariance $R$ based on $K=\mathrm{PH}^{\top}\left(\mathrm{HPH}^{\top}+\mathrm{R}\right)^{-1}$. The model error covariance $P$ is estimated based on Eq. (13).

4) Repeat the propagation and analysis steps for the next data assimilation step until the statistic convergence is reached. The observation data is used repeatedly with random observation noises at each data assimilation step.

The schematic of the algorithm for solving the acoustic inversion problem is shown in Fig. 2 . The optimal $k$ and $\varepsilon$ are inferred by assimilating the observation data with the analysis scheme of EnKF. Further, the inferred $k$ and $\varepsilon$ can be used to reconstruct the flowfield

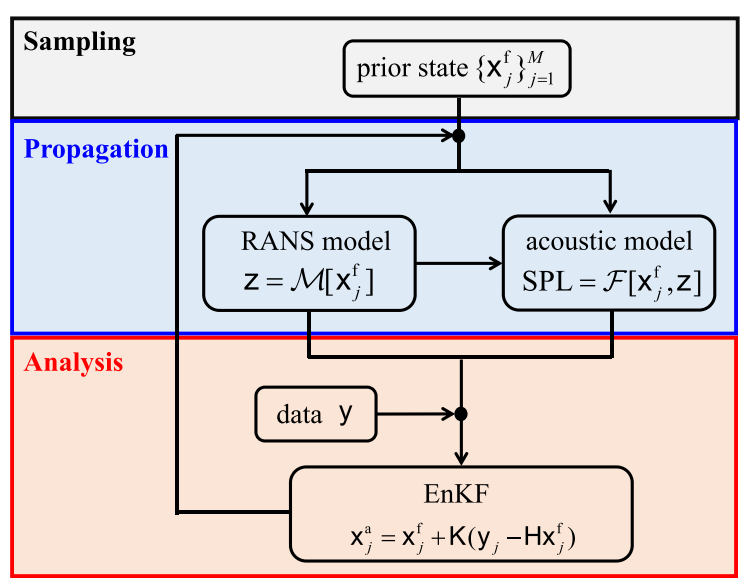

Fig. 2 Schematic of the EnKF-based acoustic inversion. 
and predict the far-field noise at unobserved locations. The proposed method is also able to simultaneously assimilate disparate data sources, for example, velocity and acoustic noise, to enhance the inference performance. It is achieved by augmenting the observation data $y$ and the error covariance $R$ with that from additional data sources [48]. The open-source code DAFI [49] is used to implement the data assimilation analysis in this work.

\section{Computational Setup}

In this work, we focus on single-stream subsonic jet flows to demonstrate the ability of the proposed EnKF-based acoustic inversion method. The method is first assessed in four cases with synthetic data and further applied to a realistic case with experimental data. For the first four test cases, the Mach number $M a$ based on the velocity $U_{b}$ at nozzle exit and the reference sound speed $340 \mathrm{~m} / \mathrm{s}$ is 0.5 . The diameter of the nozzle $D$ is $0.1016 \mathrm{~m}$, and the ratio of the temperature at the jet exit and the surrounding medium is 1 . As for the realistic case, the cold jet flow with Mach number of 0.9 and nozzle diameter of $0.05 \mathrm{~m}$ is investigated where the TKE measurements are available [50] to evaluate our inference. We use the 2-D computational domain with a wedge angle of $5 \mathrm{deg}$ to simulate the axisymmetric flow, thereby reducing the computational cost significantly. The domain and mesh grids are shown in Fig. 3 with $25 D$ in the streamwise direction and $8 D$ in the normal direction. The inlet is imposed with uniform velocity, and the axial centerline is imposed with the symmetric condition. The nozzle is imposed with no-slip conditions, the back and front patches employ the wedge boundary condition [51] by considering the round jet, and the other boundaries apply freestream conditions. The wedge boundary condition is often applied for the 2-D axisymmetric flows, specifically for a cylindrical geometry. A 2-D mesh is generated with 250 cells in the streamwise direction and 60 cells in the normal direction after grid-independent tests.

Both the axial centerline velocity and noise data are used as observations in this work. We first regard the results from compressible RANS simulations as the synthetic truth to assess the performance of the proposed framework, and the results from incompressible RANS simulations are used as the baseline. For the realistic case, the TKE measurements along the axial centerline are available from [50], and the acoustic data are available from [52]. Thus, we use the results from the compressible RANS simulation as the baseline and experimental measurements as the truth. The relative error of velocity observation is $10^{-3}$, and that of noise data is $10^{-2}$. For the noise data, we focus on the location at the observation angle of $90 \mathrm{deg}$, and thus the effects of the mean flow can be neglected. The noise spectra and noise power with the frequency bandwidth of $100 \sim 10000 \mathrm{~Hz}$ are investigated to infer the turbulence statistics. The distance between the observer and jet exit is $120 \mathrm{D}$ for the cases using synthetic data and $76 \mathrm{D}$ for the case using experimental data.

The open-source code OpenFOAM is used for numerical simulations in this work. The built-in solver simpleFoam is used to solve incompressible RANS equations, and the solver rhoSimpleFoam is used to solve compressible RANS equations. The turbulence model is the $k-\varepsilon$ model [45]. The solver rhoSimpleFoam adopts compressibility corrections on the $\varepsilon$ transport equation based on the rapid distortion theory [53] to consider the effects of the vortex stretching. Further, we build the forward solver rhonutFoam for propagation of the turbulent eddy viscosity to the velocity and density by solving the RANS equations. Specifically, the given TKE and dissipation rate are first used to compute the eddy viscosity based on $\nu_{t}=C_{\mu}\left(k^{2} / \varepsilon\right)$, where $C_{\mu}=0.09$, and the forward solver propagates the computed eddy viscosity $\nu_{t}$ to the velocity and density through solving compressible RANS equations.

To verify our implementation, we compare the jet noise prediction based on Tam and Auriault's method with the experimental data from Ref. [14]. The case setup is the same with the round jet of $M a=0.5$ and $\bar{D}=0.1016 \mathrm{~m}$ as illustrated before. The compressible solver rhoSimpleFoam is used to compute the mean flowfield and the TKE and dissipation rate, and Tam and Auriault's model is used to predict the far-field noise spectra. The acoustic model parameters are tuned to be $A=8, c_{l}=0.25, c_{\tau}=0.1$ based on experimental data. The results of the mean flow solution and the far-field noise prediction are shown in Fig. 4. It is obvious that Tam and Auriault's model can predict the far-field noise in good agreement with the reference data.

As for the data assimilation setup, both the variance $\sigma$ and characteristic length $l$ in the kernel function (11) are chosen as constant 0.1 in this work. The sensitivity study of the parameters $\sigma$ and $l$ is provided in the Appendix. We use the first 100 modes to cover more than $99 \%$ of variance and generate 50 samples to estimate the ensemble covariance. The plots of the baseline $k$ and $\varepsilon$ and the relative discrepancy for the cases using synthetic data are shown in Fig. . The sample means almost overlap with the baseline, which is reasonable since the prior samples regard the baseline results as the mean. It is noticeable that the TKE and dissipation rate are slightly smaller than those from the compressible solver, likely due to the neglect of dilatation effects in the baseline simulation with the incompressible solver.

We use five cases with different observations and inferred quantities to gradually illustrate the ability of the proposed framework for the acoustic inversion. The details of the case setup are summarized in Table 1 . In the first case, we infer the TKE and dissipation rate from the axial centerline velocity. In the second case, we use the acoustic noise measurements (e.g., the noise spectra and noise power) to identify the noise sources. Both the velocity and the noise data can be measured with experimental techniques such as PIV and microphones. The noise measurements are the integral data source, while the velocity data are the volume data source, which are disparate. It has been demonstrated that the assimilation of the disparate data sources is able to improve the mean flow reconstruction [48]. Hence, in the third case, we investigate the inference of TKE and dissipation rate with the observation of both velocity and noise to improve the estimation of near-field flow and far-field noise. The preceding cases

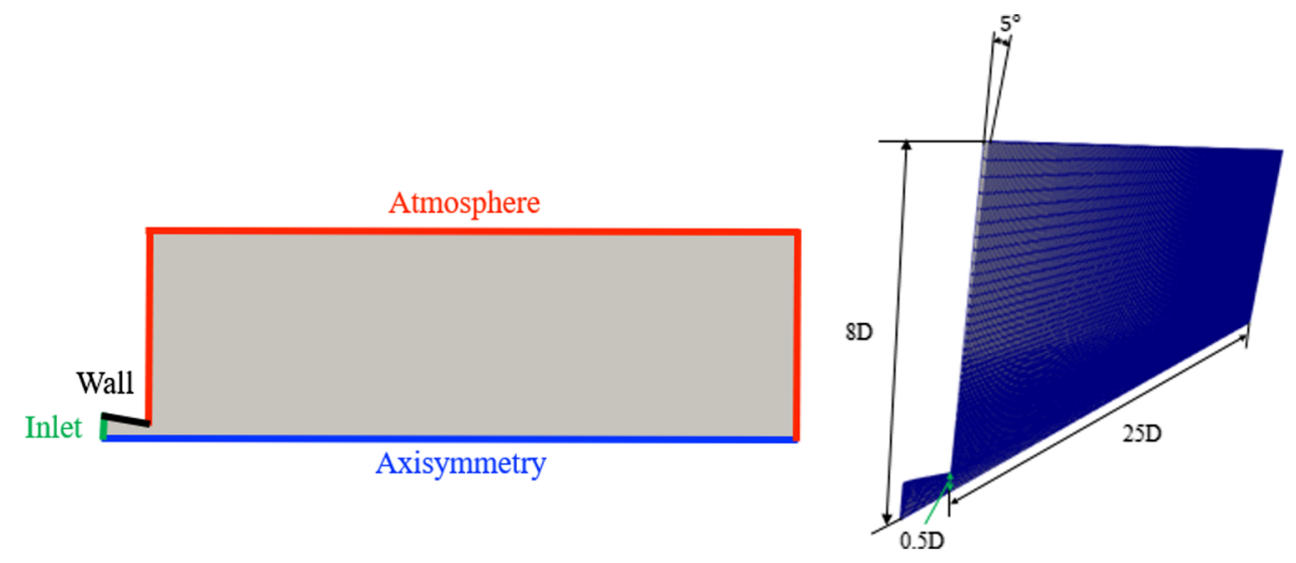

a) Computational domain

b) Mesh grids

Fig. 3 Computation domain and mesh grids. 


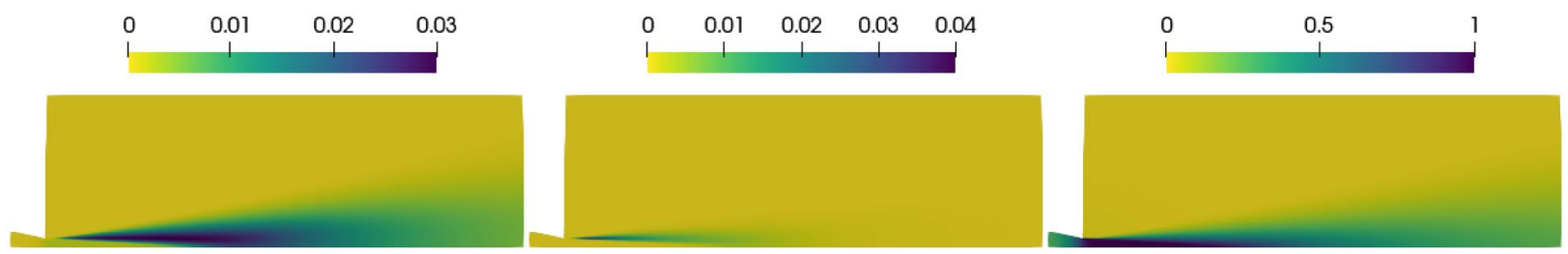

a) TKE $\left(k / U_{b}^{2}\right)$

b) dissipation rate $\left(\varepsilon D / U_{b}^{3}\right)$

c) Velocity $\left(U_{1} / U_{b}\right)$

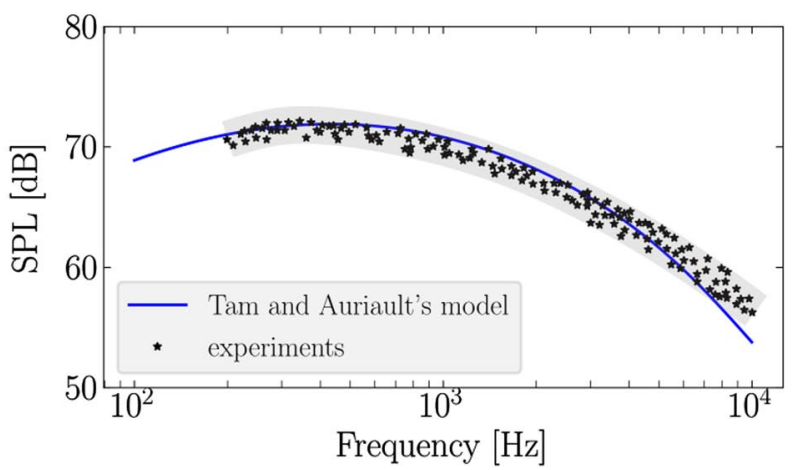

d) noise spectrum

Fig. 4 Prediction of the mean flows and far-field noise spectrum at the $90 \mathrm{deg}$ observer angle.

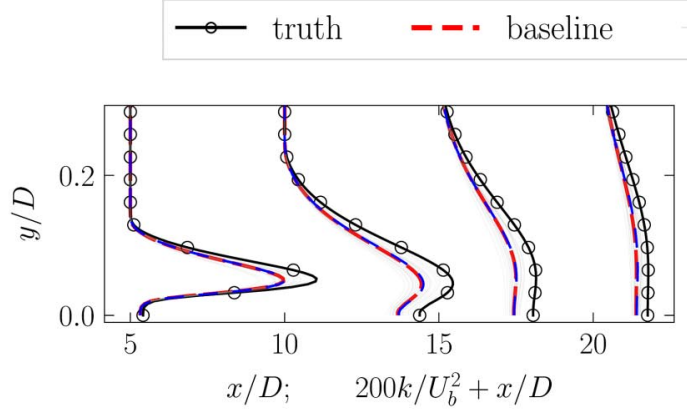

a) $k$

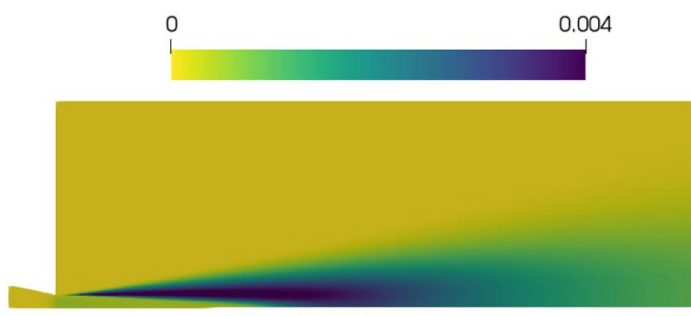

c) relative discrepancy of $k$

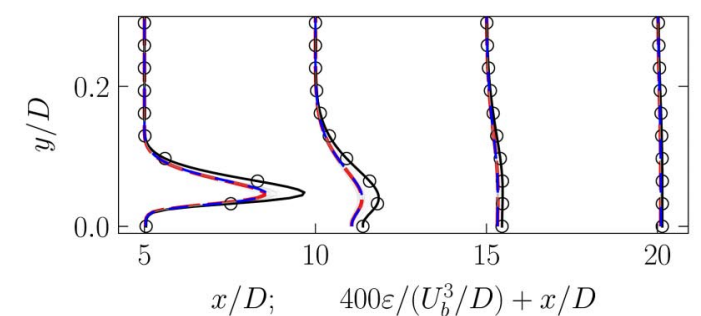

b) $\varepsilon$

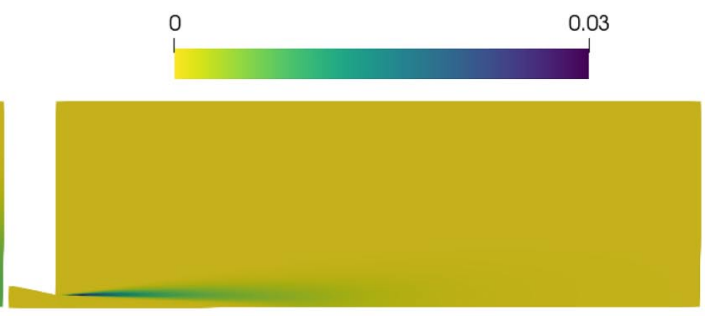

d) relative discrepancy of $\varepsilon$

Fig. 5 Comparison plots of the TKE $k$ and dissipation rate $\varepsilon$ among the truth, the baseline, and the initial samples. The contour plots indicate the relative discrepancy of the inferred $q$ with respect to the truth $\left|q-q^{\text {truth }}\right| /\left\|q^{\text {truth }}\right\|$.

explore the effects of different observations on the inference where the acoustic model parameters are determined as a priori. In the fourth case, we use the noise and velocity data to infer the $k$ and $\varepsilon$ and

\section{Table 1 Summary of inference setup for test cases}

\begin{tabular}{lccc}
\hline \hline Case & Observation data, $\mathrm{y}$ & Dim, $\mathrm{y}$ & Inferred quantities, $\mathbf{x}$ \\
\hline 1 & $U_{1}$ & 250 & $\boldsymbol{w}^{k}, \boldsymbol{w}^{\varepsilon}$ \\
$2 a$ & $\mathrm{SPL}$ & 200 & $\boldsymbol{w}^{k}, \boldsymbol{w}^{\varepsilon}$ \\
$2 b$ & $\mathrm{I}$ & 1 & $\boldsymbol{w}^{k}, \boldsymbol{w}^{\varepsilon}$ \\
$3 a$ & $U_{1}, \mathrm{SPL}$ & 450 & $\boldsymbol{w}^{k}, \boldsymbol{w}^{\varepsilon}$ \\
$3 b$ & $U_{1}, \mathrm{I}$ & 251 & $\boldsymbol{w}^{k}, \boldsymbol{w}^{\varepsilon}$ \\
4 & $U_{1}, \mathrm{SPL}$ & 450 & $\boldsymbol{w}^{k}, \boldsymbol{w}^{\varepsilon}, A, c_{l}, c_{\tau}$ \\
5 & $U_{1}, \mathrm{SPL}$ & 38 & $\boldsymbol{w}^{k}, \boldsymbol{w}^{\varepsilon}, A, c_{l}, c_{\tau}$ \\
\hline \hline
\end{tabular}

simultaneously determine the empirical parameters in the acoustic model. In the final case, we apply the proposed acoustic inversion framework in a realistic application where the experimental measurements of noise spectra, mean velocity, and TKE are available.

\section{Inference Results}

\section{A. Assimilation of Velocity}

As the first case, we use the velocity observation to reconstruct the flowfield, which is commonly investigated in existing literature $[23,24]$. Here we focus on using the velocity data along the axial centerline to improve the noise prediction. The data assimilation results of the TKE $k$ and dissipation rate $\varepsilon$ are shown in Fig. 6. It can be seen that the velocity data improve the inference of TKE and dissipation rate, but noticeable discrepancies still exist. That is likely because the velocity is related to the eddy viscosity in the RANS 


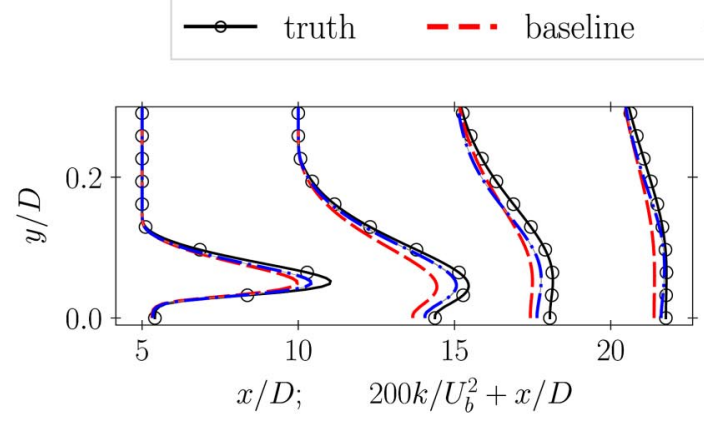

a) TKE $k$

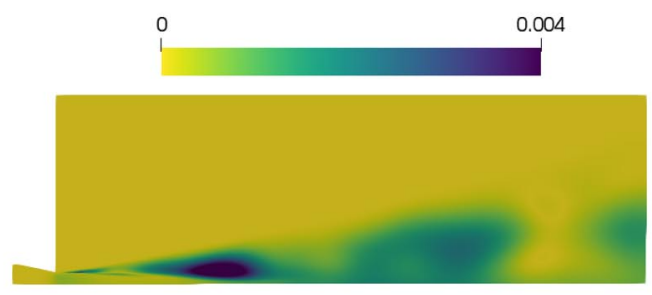

c) discrepancy of $k$ samples $\quad-\quad$ sample mean

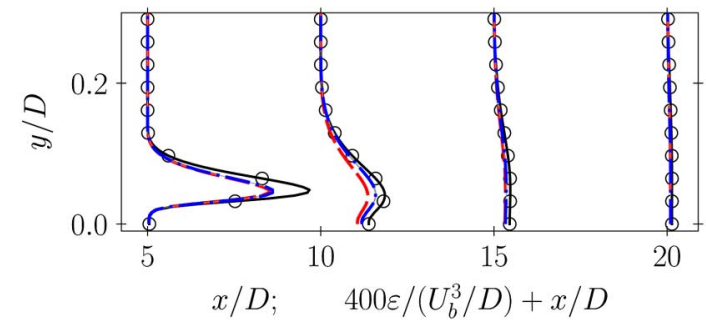

b) dissipation rate $\mathcal{E}$

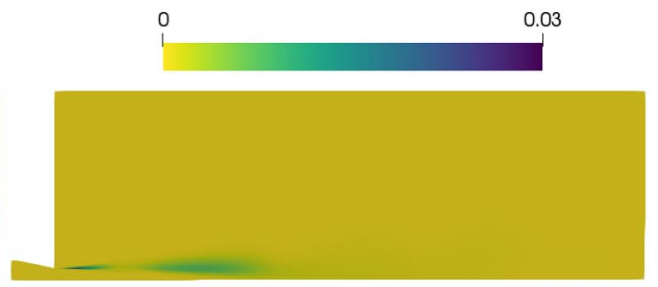

d) discrepancy of $\varepsilon$

Fig. 6 Inferred $k$ and $\varepsilon$ (the posterior sample mean) by assimilating the observation of axial centerline velocity for case 1 . The contour plots indicate the relative discrepancy of the inferred quantity $q$ with respect to the truth $\left|q-q^{\text {truth }}\right| /\left\|q^{\text {truth }}\right\|$.

equations. Using the velocity data, it is possible to infer the eddy viscosity, particularly in the region where the mean strain rate is large, but it is difficult to infer $k$ and $\varepsilon$ since the eddy viscosity is a combination of the TKE and dissipation rate.

Figure 7 presents the predicted velocity and far-field noise spectra with the inferred $k$ and $\varepsilon$. The magnitudes of the velocity are slightly smaller than the baseline results from incompressible RANS simulation. That is probably due to the fact that the incompressible RANS method does not consider the compressibility effects and ignores the dilatation dissipation. That would underestimate the turbulence dissipation and lead to the relatively slow reduction of the velocity compared to the synthetic truth. It can be seen that the reconstructed velocity has significant improvements in the entire computational domain after the assimilation of centerline velocity data. On the other hand, with the inferred TKE and dissipation rate, the far-field noise can be predicted as shown in Figs. 7c and 7d. The prediction in SPL is improved slightly but still exhibits large discrepancies compared to synthetic truth, as well as at the locations of $r=60 D$ and $r=200 D$. Hence, the velocity data are effective to improve the reconstruction of full-field velocity but are limited for the inference of the TKE and dissipation rate.

The errors in the inference of the $k$ and $\varepsilon$ and the prediction of the $U_{1}$ and SPL are summarized in Table 2 . The errors for each quantity $q$ over the entire computational domain are computed based on

$$
\text { error }=\frac{\left\|q^{\text {estimate }}-q^{\text {truth }}\right\|}{\left\|q^{\text {truth }}\right\|}
$$

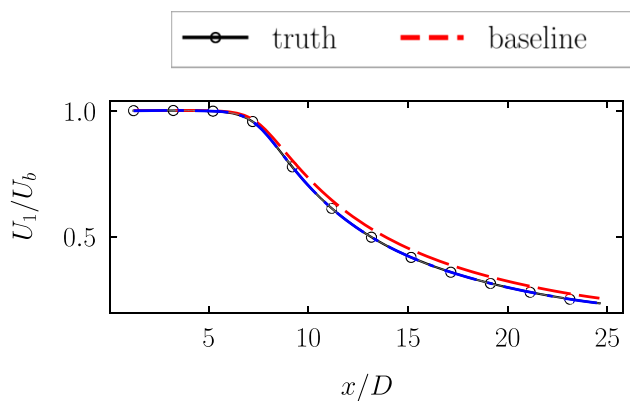

a) axial centerline velocity

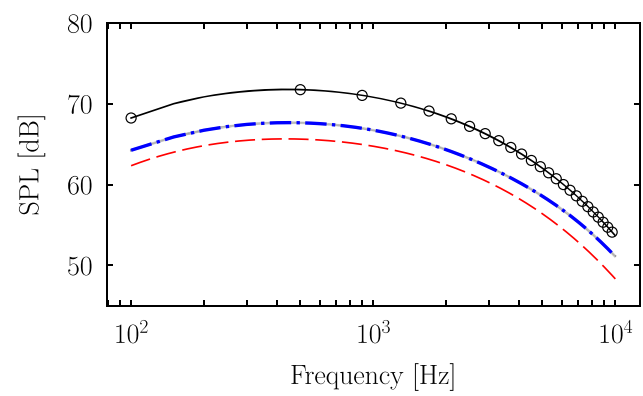

c) SPL at $r=120 \mathrm{D}$ samples $\quad-\quad$ sample mean

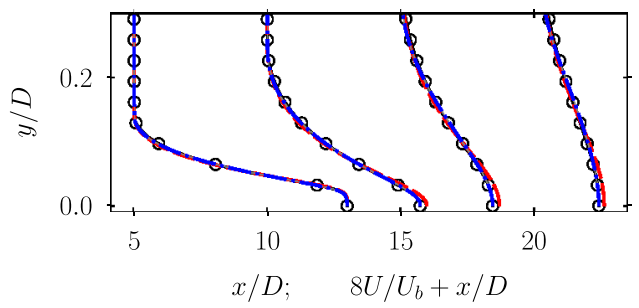

b) velocity profiles

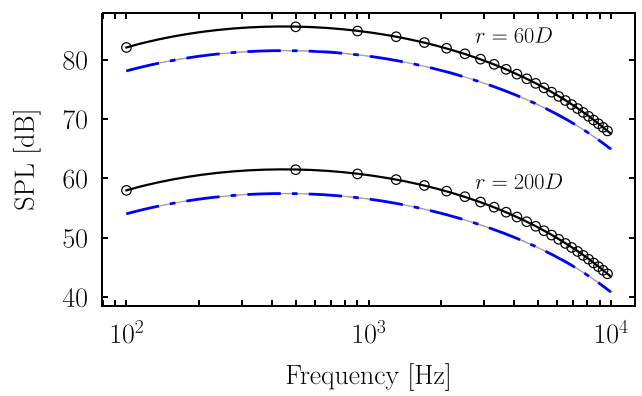

d) SPLs at $r=60 D$ and $r=200 D$

Fig. 7 Reconstructed velocity and noise spectra (the posterior sample mean) by assimilating the observation of axial centerline velocity for case 1. 
Table 2 Summary of the prediction error in the velocity field $U$, the noise spectra SPL, and the TKE $k$ and dissipation rate $\varepsilon$ for synthetic cases 1-4. The error is estimated based on Eq. (17)

\begin{tabular}{lrlrc}
\hline \hline Case & err, $U$ & err, SPL & \multicolumn{1}{c}{ err, $k$} & err, $\varepsilon$ \\
\hline Baseline & $3.49 \%$ & $9.07 \%$ & $19.34 \%$ & $26.35 \%$ \\
1 & $1.35 \%$ & $5.54 \%$ & $14.12 \%$ & $20.08 \%$ \\
$2 a$ & $4.31 \%$ & $0.0038 \%$ & $9.30 \%$ & $14.91 \%$ \\
$2 b$ & $11.01 \%$ & $2.48 \%$ & $23.62 \%$ & $21.04 \%$ \\
$3 a$ & $2.08 \%$ & $0.021 \%$ & $13.18 \%$ & $13.81 \%$ \\
$3 b$ & $2.93 \%$ & $0.30 \%$ & $13.57 \%$ & $17.64 \%$ \\
4 & $1.21 \%$ & $0.010 \%$ & $14.03 \%$ & $26.02 \%$ \\
\hline \hline
\end{tabular}

It clearly shows that the reconstructed noise spectrum is improved in contrast to the baseline but still exhibits the considerable discrepancies of $5.54 \%$, while the discrepancy of the velocity field can be reduced to around $1.35 \%$. On the other hand, both the inferred $k$ and $\varepsilon$ are improved slightly compared to the baseline.

\section{B. Assimilation of Far-Field Noise}

In this subsection, we employ the proposed acoustic inversion framework to infer the turbulent kinetic energy and dissipation rate from the measurements of noise spectra or noise power. We first investigate the use of the noise spectra to infer the noise sources. Figure 8 shows the plots of the inferred TKE and dissipation rate based on the observation of the noise spectra. It can be seen that assimilating the noise spectra improves the estimation of $k$ and $\varepsilon$ significantly. The TKE is recovered accurately near the jet nozzle but underestimated relatively at the downstream. That is because the high-frequency noise is produced by the small turbulent eddies near the nozzle. Accordingly, the noise spectra at the high-frequency range are able to enforce the inference of the noise source near the nozzle. However, the low-frequency noise is mainly generated by the large-scale eddies in the downstream but also contributed by the turbulence in the relative upstream regions. This leads to the conclusion that the noise sources at the downstream are not sensitive to the noise spectra. Hence, the reconstructed TKE is improved mainly near the nozzle rather than downstream by incorporating the observation of noise spectra.

The reconstructed velocity with the inferred $k$ and $\varepsilon$ is shown in Fig. 9. It is obvious that the velocity field agrees with the observation data near the jet nozzle exit but exhibits noticeable discrepancies at the downstream. That is likely due to the discrepancies in the TKE and dissipation rate at the downstream, leading to the discrepancy in eddy viscosity and further in velocity. The predicted SPL spectrum is provided in Fig. 9, showing a remarkable agreement with the reference data. Although the inferred quantities exhibit discrepancies, the noise spectra still agree with the synthetic truth. This shows that the acoustic inversion problem is highly ill-posed. That is, various fields of TKE and dissipation rate result in the similar far-field noise prediction. In spite of the inference discrepancies, the inferred field can be assumed as optimal corrections for the low-order noise models. It is useful to aid the jet noise modeling and improve the noise estimation at unobserved locations as shown in Fig. 9.

The errors in the inference and prediction are presented in Table 2. In contrast to the velocity, assimilation of the noise measurement can improve the inference in both $k$ and $\varepsilon$ and significantly enhance the prediction of the noise spectra. Specifically, the discrepancy in the noise spectra reduces to around $0.00382 \%$ from $9.07 \%$ of the baseline. The discrepancies of $k$ and $\varepsilon$ are reduced to $9.3 \%$ and $14.9 \%$, respectively, compared to $19.3 \%$ and $26.4 \%$ of the baseline.

Further, we investigate using noise power I to infer the jet noise source. The noise power is the integration of noise intensity given a bandwidth of frequency, which is relatively straightforward to be obtained compared to the noise spectra. Hence, it is worthy of investigation to use the noise power measurement for inversion of the noise sources. We use the noise power with a bandwidth of $100 \sim 10000 \mathrm{~Hz}$. However, the inferred TKE and dissipation rate have considerable discrepancies. The plots of data assimilation results are omitted for brevity, and the error estimation is summarized in Table 2. The large discrepancy leads to the conclusion that both the predicted noise spectra and flowfield are worse than the other test cases. The noise power is able to match with the reference data, while the spectra (i.e., the distribution of the acoustic energy in the frequency) cannot be recovered with such limited information.

\section{Assimilation of Velocity and Far-Field Noise}

In the preceding two test cases, the velocity data are able to improve the reconstruction of the velocity field but cannot reconstruct well the fields of TKE and dissipation rate. In contrast, the acoustic noise data can improve the reconstruction of the TKE and dissipation rate but lead to large discrepancies in the reconstructed velocity field. Hence, in this section we integrate the velocity and far-field noise data to enhance the inference of the noise sources. The data assimilation results in TKE and dissipation rate are shown in Fig. 10. It shows that the TKE and dissipation rate are obviously improved, but the discrepancies still exist mainly at the downstream, as in case $2 a$.

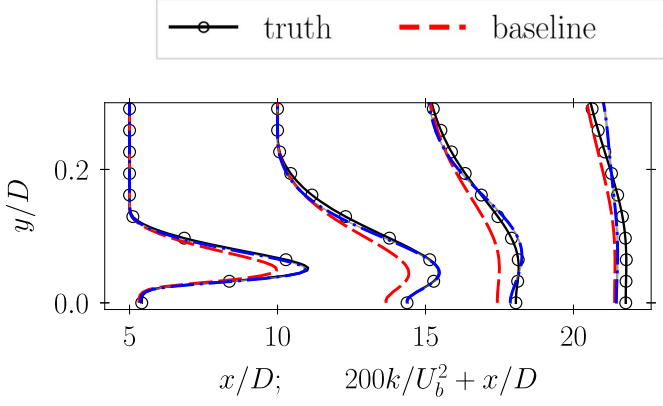

a) TKE $k$

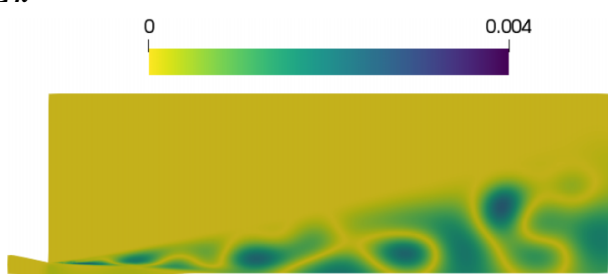

c) discrepancy of $k$ samples $\quad-\quad-$ sample mean

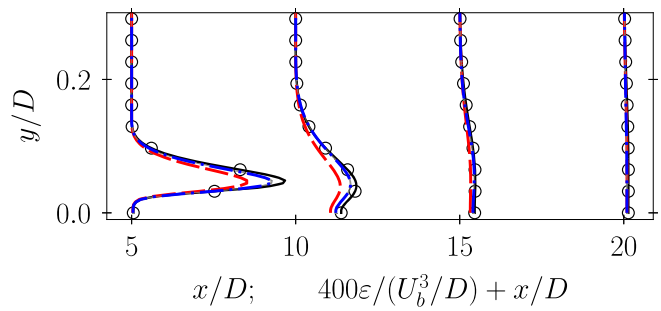

b) dissipation rate $\varepsilon$

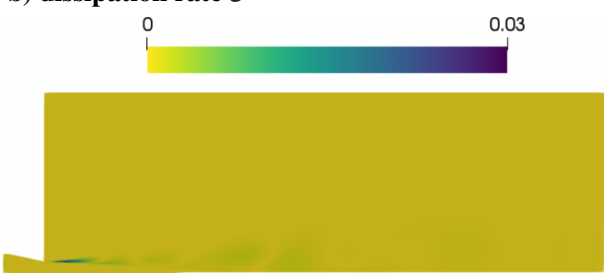

d) discrepancy of $\varepsilon$

Fig. 8 Inferred $k$ and $\varepsilon$ (the posterior sample mean) by assimilating the observation of noise spectrum at $120 D$ for case $2 a$. The contour plots indicate the relative discrepancy of the inferred quantity $q$ with respect to the truth $\left|q-q^{\text {truth }}\right| /\left\|q^{\text {truth }}\right\|$. 


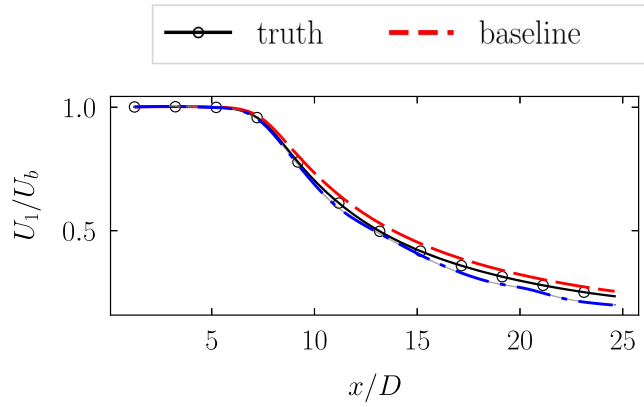

a) axial centerline velocity

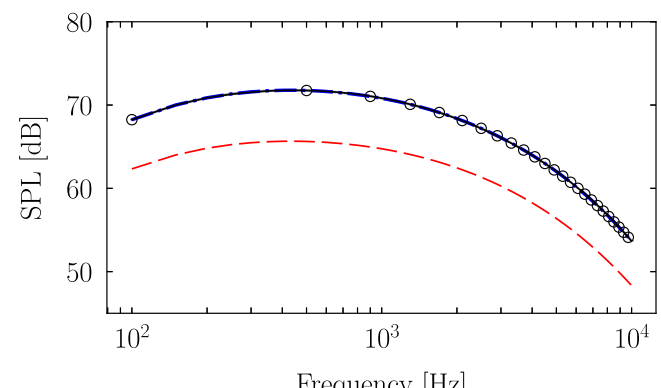

c) SPL at $r=120 D$ samples $\quad-\quad-$ sample mean

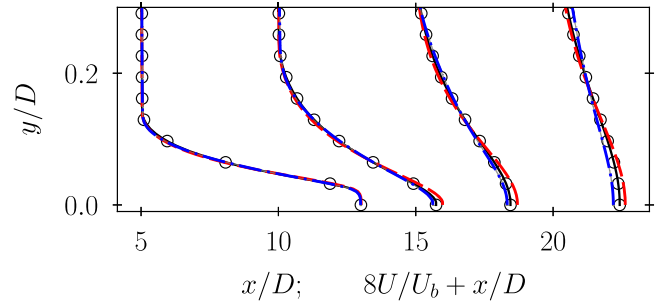

b) velocity profiles

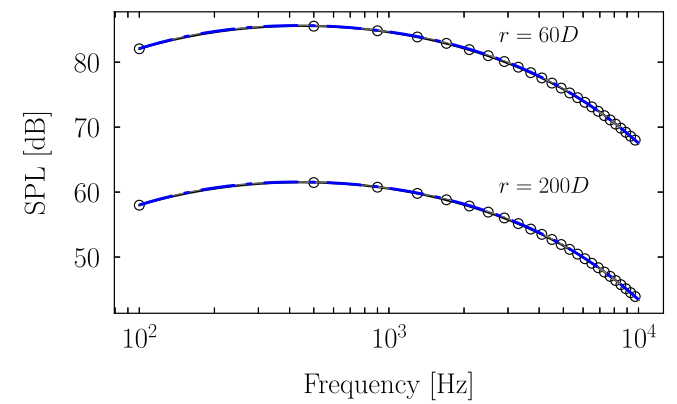

d) SPLs at $r=60 D$ and $r=200 D$

Fig. 9 Reconstructed velocity and noise spectra (the posterior sample mean) by assimilating the observation of axial centerline velocity for case $2 a$.

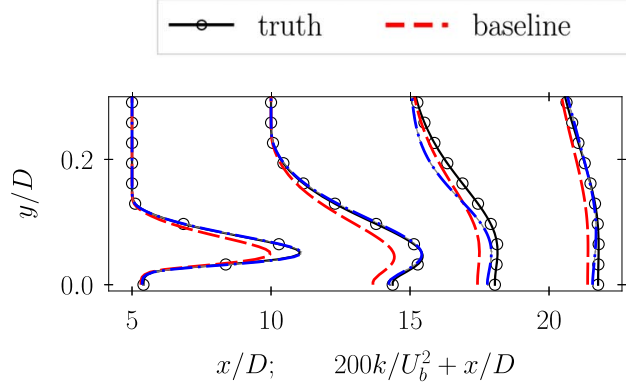

a) $k$

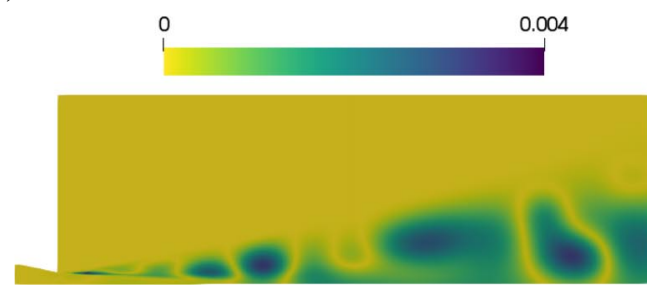

c) discrepancy of $k$ samples $\quad-\quad-$ sample mean

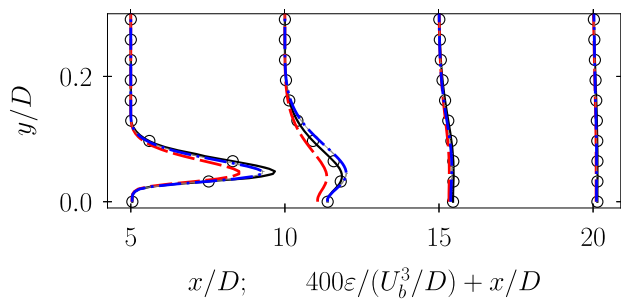

b) $\mathcal{E}$

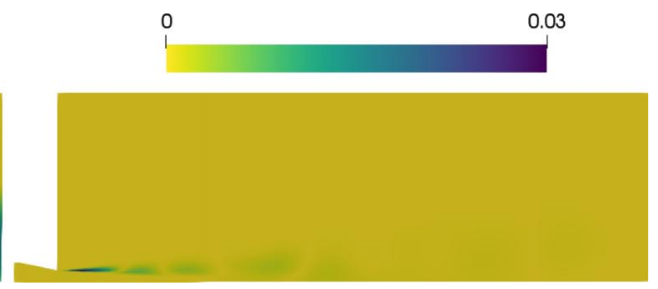

d) discrepancy of $\varepsilon$

Fig. 10 Inferred $k$ and $\varepsilon$ fields (the posterior sample mean) by assimilating the observation of axial centerline velocity and noise spectrum at $120 D$ for case $3 a$. The contour plots indicate the relative discrepancy of the inferred quantity $q$ with respect to the truth $\left|q-q^{\text {truth }}\right| /\left\|q^{\text {truth }}\right\|$.

Figure 11 shows the results of the predicted velocity and noise spectra. Both velocity and noise spectra are reconstructed accurately with the inferred TKE and dissipation rate. In contrast to the assimilation of only the velocity or the noise measurements in cases $2 a$ or $2 b$, simultaneously incorporating the noise measurement and velocity can improve the reconstruction of both the near-field velocity and the far-field noise spectra. The TKE and dissipation rate have noticeable discrepancies particularly at the downstream, but the noise spectra can still be well reconstructed. That is due to the same reason as in case $2 a$; that is, the turbulence at the downstream makes limited contributions to the overall noise spectra.

The estimated errors of the inference and the prediction are summarized in Table 2. It can be seen that the errors of both velocity and acoustic noise are reduced. However, the reconstructed velocity is less accurate compared to case 1 , where only axial centerline velocity is assimilated, and the inferred $k$ is slightly inferior to case $2 a$, where only noise spectra are assimilated. That is likely due to the fact that this case aims to reduce the discrepancy in the combination of the velocity and noise spectra.

In case $2 b$, it has been observed that the velocity and the noise power cannot provide satisfactory reconstruction of the flowfield and the noise spectra. To this end, we further investigate the assimilation of the measurements integrating axial centerline velocity and acoustic noise power. We use the noise power with the same bandwidth in case $2 b$ and the axial centerline velocity to infer the TKE $k$ and dissipation rate $\varepsilon$. The inferred TKE and dissipation rate are shown in Fig. 12, and noticeable improvements in the inference of turbulence quantities are achieved through combining the velocity and noise 


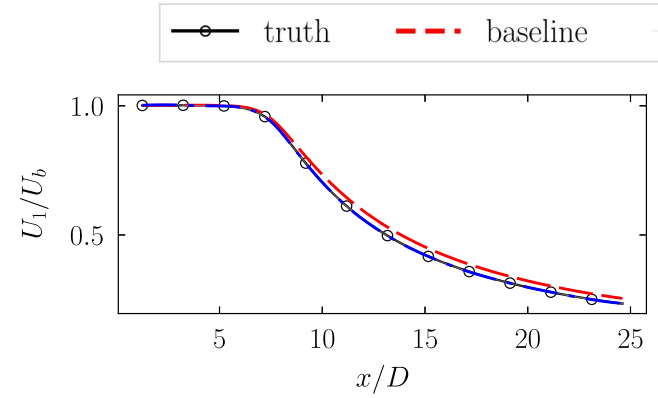

a) axial centerline velocity

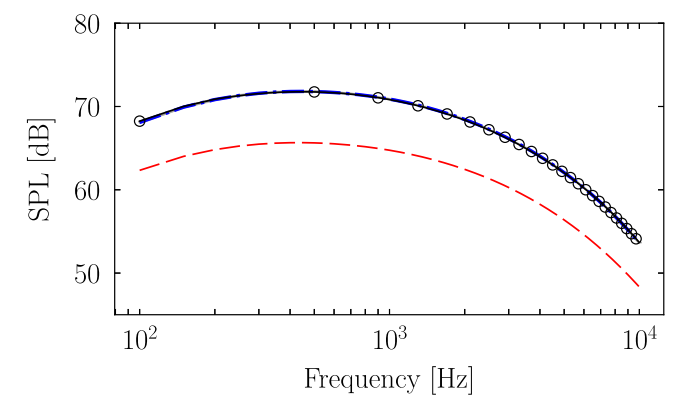

c) SPL at $r=120 D$ samples $\quad-\quad$ sample mean

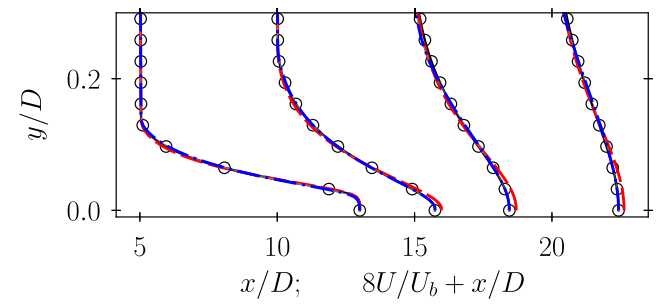

b) velocity profiles

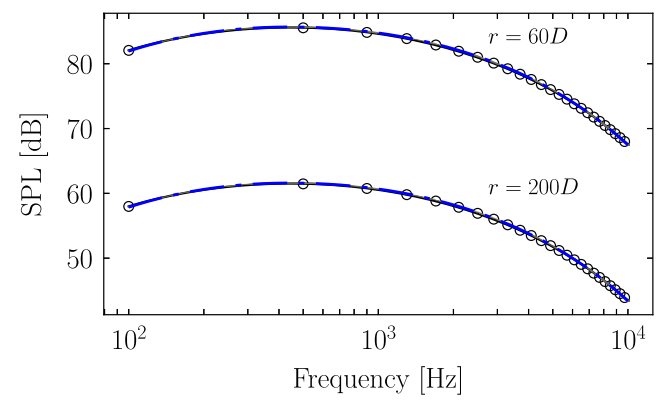

d) SPLs at $r=60 D$ and $r=200 D$

Fig. 11 Reconstructed velocity and noise spectra (the posterior sample mean) by assimilating the observation of axial centerline velocity and noise spectrum at $120 D$ for case $3 a$.

power data. With the inferred $k$ and $\varepsilon$, the velocity and noise spectra can be reconstructed as presented in Fig. 13. It shows that the velocity remarkably agrees with the reference data, and the noise spectra also fit well with the reference except in the low-frequency range. The proposed method is able to improve the estimation of both the velocity field and the noise spectra by assimilating the observation of axial centerline velocity and noise power.

\section{Inference of TKE, Dissipation Rate, and Acoustic Model Parameters}

In the preceding subsections, we assess the performance of different observation data for inferring the TKE and dissipation rate, demonstrating that integrating the observation of velocity and noise spectra can improve the reconstruction of both velocity and noise spectra. However, the noise model uncertainty in the empirical parameters of the space-time correlation function is not considered and determined as a priori. In this subsection, we consider the uncertainty from the RANS model in the TKE and dissipation rate and that from the noise model in the empirical parameters $A$, $c_{l}, c_{\tau}$. The baseline values of acoustic model parameters $A, c_{l}, c_{\tau}$ are chosen as $8,0.2,0.2$, which is in consistent with those in Ref. [54]. The reference values of these parameters have been given in Sec. III as $8,0.25,0.1$. We augment the parameters in the state so that $\mathbf{x}=\left[\boldsymbol{w}^{k}, \boldsymbol{w}^{\varepsilon}, A, c_{l}, c_{\tau}\right]$. The standard deviation of each parameter is $2,0.1,0.1$, respectively. The sensitivity study of the standard deviation for each parameter is provided in the Appendix. Other setup is in consistent with previous cases. Based on the observation of velocity and noise spectrum, we infer the

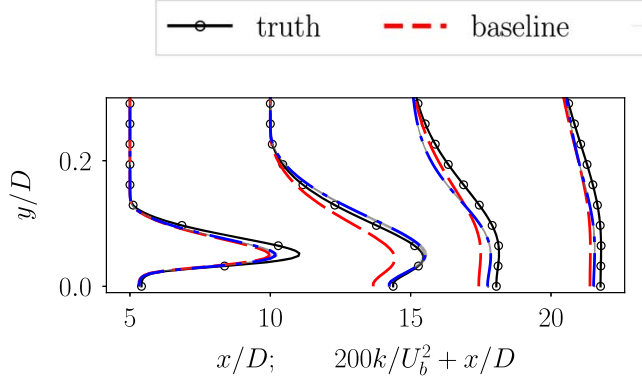

a) $k$

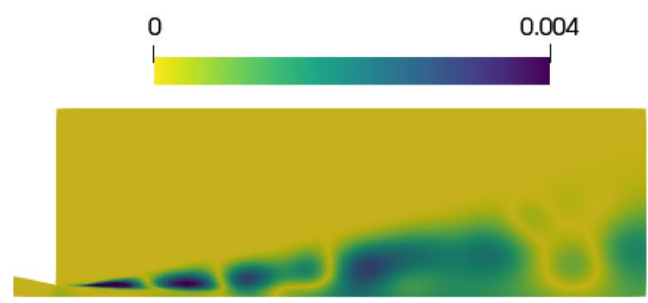

c) discrepancy of $k$

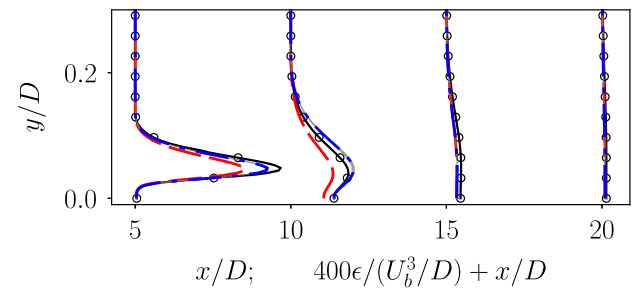

b) $\varepsilon$
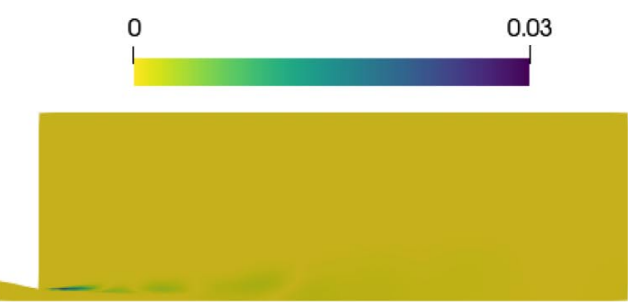

d) discrepancy of $\varepsilon$

Fig. 12 Inferred $k$ and $\varepsilon$ (the posterior sample mean) by assimilating the observation of axial centerline velocity and noise spectrum at $120 D$ for case $3 b$. The contour plots indicate the relative discrepancy of the inferred quantity $q$ with respect to the truth $\left|q-q^{\text {truth }}\right| /\left\|q^{\text {truth }}\right\|$. 


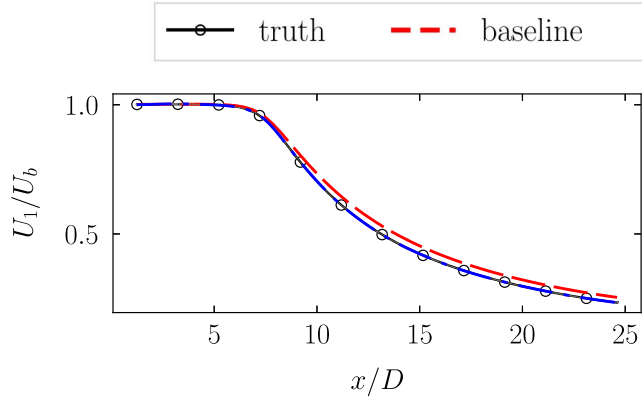

a) axial centerline velocity

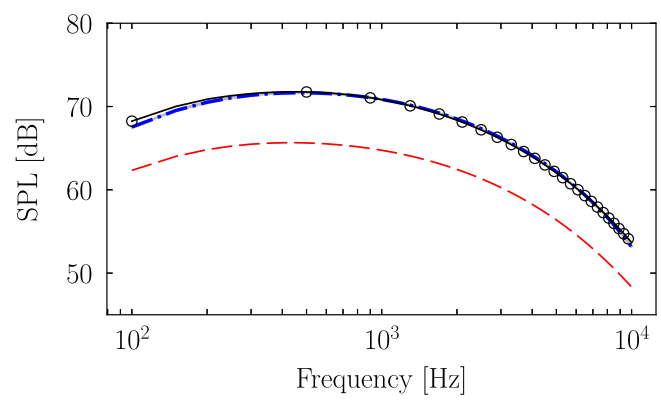

c) SPL at $r=120 D$ samples - - sample mean

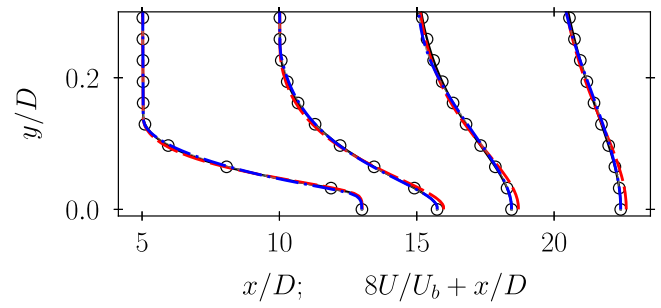

b) velocity profiles

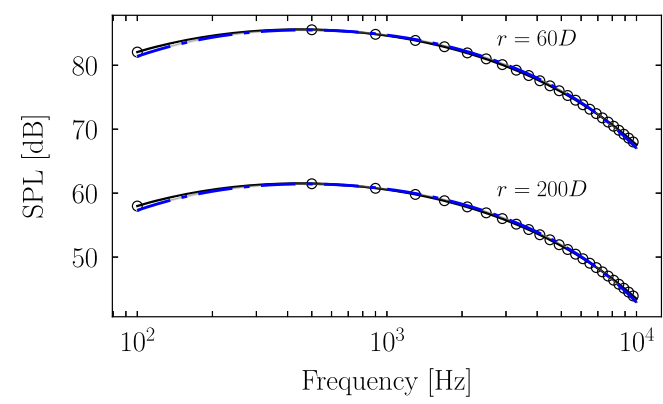

d) SPLs at $r=60 D$ and $r=200 D$

Fig. 13 Reconstructed velocity and noise spectra (the posterior sample mean) by assimilating the observation of axial centerline velocity and noise power for case $3 b$.

noise model parameters, turbulent kinetic energy, and dissipation rate simultaneously.

The reconstructed velocity and spectrum can have good agreements with the reference data as shown in Fig. 14. The plots of the inferred parameters are shown in Fig. 15. It can be seen that the inferred parameter $A$ converges to around 10 and has large departures from the synthetic truth, while both the parameters $c_{l}$ and $c_{\tau}$ are improved compared to the baseline. Particularly, the inferred parameter $c_{\tau}$ has good alignments with the reference. The plots of the inferred TKE and dissipation rate are shown in Fig. 16. Clearly, limited improvements are achieved in the turbulent kinetic energy, while the dissipation rate is almost unchanged compared to the baseline. Even though the turbulent kinetic energy, the dissipation rate, and the noise model parameters cannot be inferred accurately, the constructed velocity and noise spectrum are in good alignment with the observations. That is because the uncertainty of the RANS model and noise model is not separated during the assimilation process. Specifically, the underestimated TKE and the overestimated parameter $A$ could be combined to provide satisfactory results in noise spectra and velocity fields. To further improve the inference,

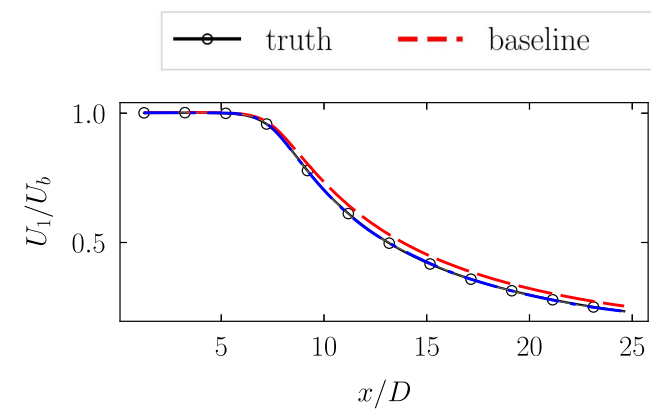

a) axial centerline velocity

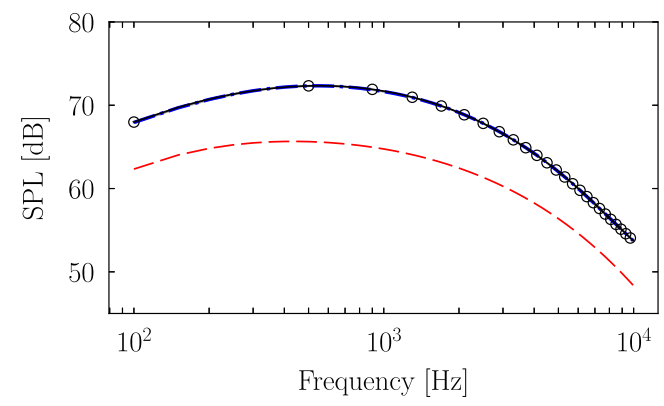

c) SPL at $r=120 D$ samples $\quad-\quad$ sample mean

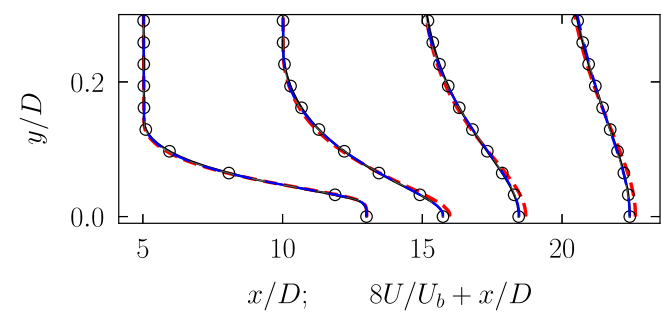

b) velocity profiles

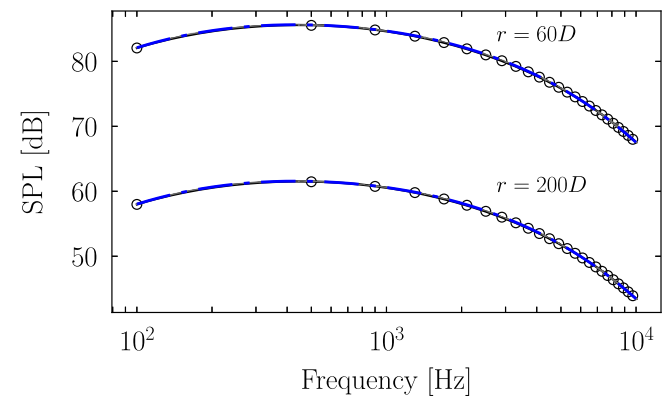

d) SPLs at $r=60 D$ and $r=200 D$

Fig. 14 Reconstructed velocity and noise spectra (the posterior sample mean) by assimilating the observation of axial centerline velocity and noise power for case 4 . 


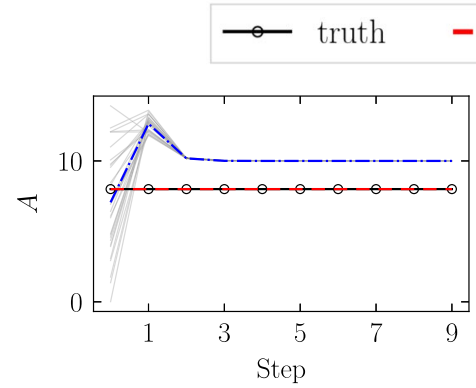

a) parameter $A$
- - - baseline

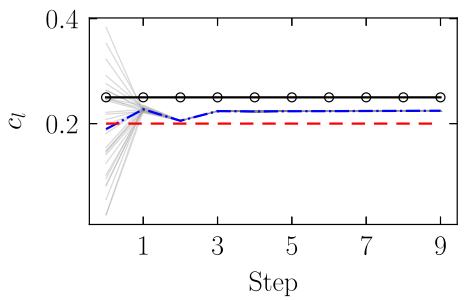

b) parameter $c_{l}$ samples $\quad-\quad$ sample mean

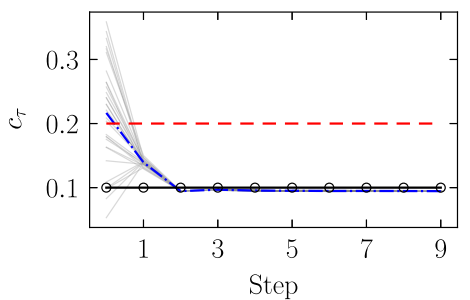

c) parameter $c_{\tau}$

Fig. 15 Convergence plots of the acoustic model parameters $A, c_{l}$, and $c_{\tau}$ for case 4 . The inferred parameters are indicated by the posterior sample mean.

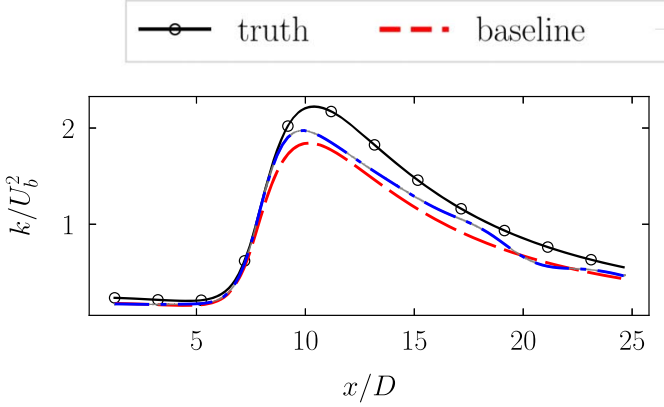

a) axial centerline TKE $k$

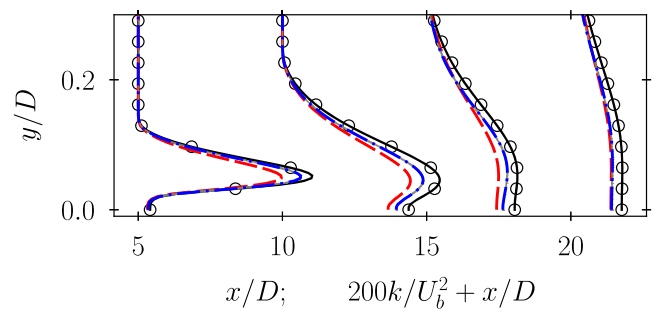

(c) TKE $k$ along profiles samples $\quad-\quad$ sample mean

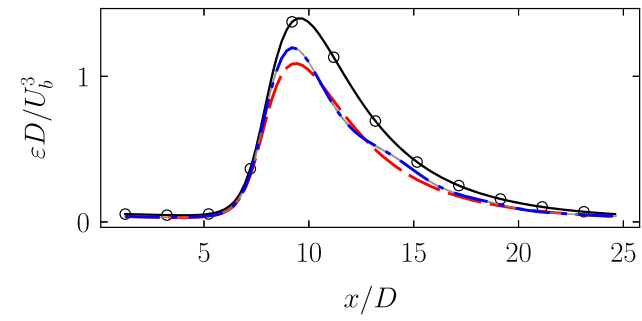

b) axial centerline dissipation rate $\varepsilon$

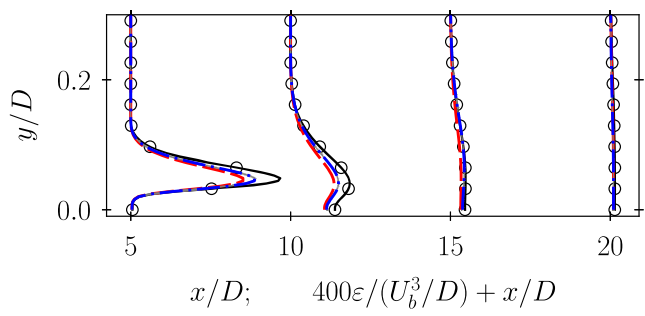

d) dissipation rate $\varepsilon$ along profiles

Fig. 16 Inferred TKE $k$ and dissipation rate $\varepsilon$ (the posterior sample mean) by assimilating the observation of axial centerline velocity and noise spectrum at $r=120 D$ for case 4 .

additional observation data, for example, noise spectra at other observer angles, are needed to constrain the inference process. The errors of inference and prediction are summarized in Table 2, clearly showing the error reduction in velocity, SPL spectra, and TKE.

\section{E. Realistic Case}

Finally, we employ the proposed EnKF-based acoustic inversion method in a realistic application. The jet flow with Mach number of 0.9 and the nozzle diameter $D$ of $0.05 \mathrm{~m}$ is investigated. The experimental data are available from Ref. $[\underline{50}, \underline{55}]$ and have been widely used for numerical validations $[18,52]$. As in case 4 , we consider the uncertainty in the TKE and dissipation rate as well as the empirical parameters $A, c_{l}, c_{\tau}$ in the space-time correlation function. We use the $A=8, c_{l}=0.2, c_{\tau}=0.2$ as the baseline parameters and the RANS results from the compressible solver as the baseline for the TKE and dissipation rate. Other setup is in consistent with the previous cases.

The inferred parameters are shown in Fig. 17, converging to around $A=5.10, c_{l}=0.05, c_{\tau}=0.07$. By considering the sparse observation of the axial centerline velocity and the noise spectrum, we can reconstruct the noise spectrum and the velocity as shown in Figs. $18 \mathrm{a}$ and $\underline{18 \mathrm{~b}}$, where the Strouhal number $S t$ is based on the

$$
\text { - - - baseline } \quad \text { samples } \quad-\text { sample mean }
$$

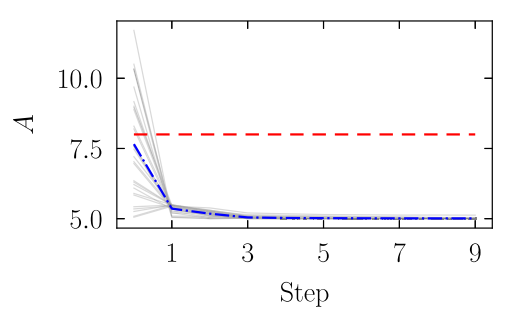

a) parameter $A$

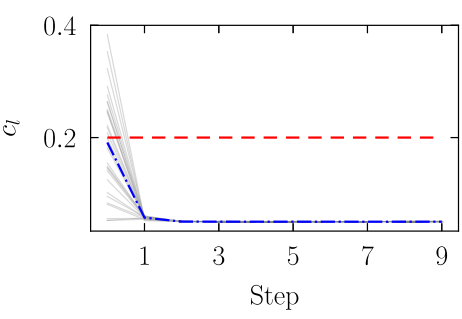

b) parameter $c_{l}$

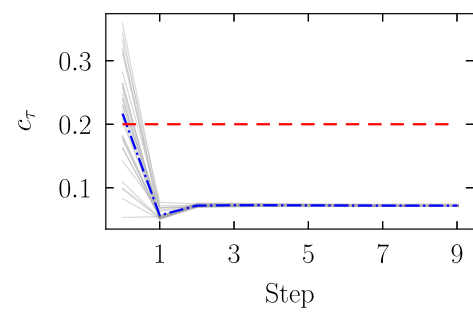

c) parameter $c_{\tau}$

Fig. 17 Convergence plots of the acoustic model parameters for the realistic case 5 . The inferred parameters are indicated by the posterior sample mean. 


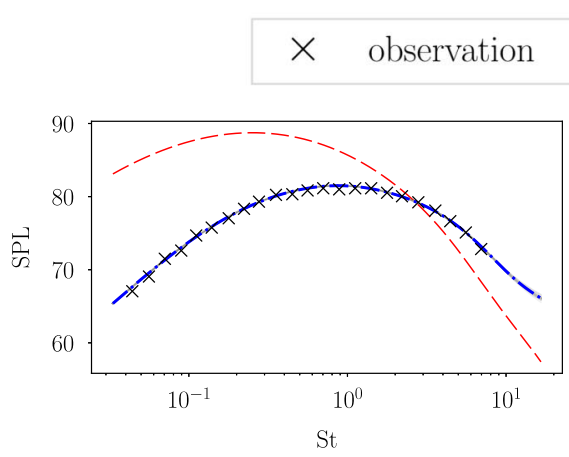

a) SPL spectrum

- - baseline $\quad$ sample - - s sample mean

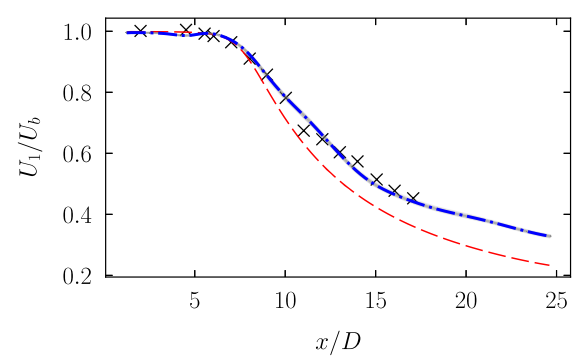

b) axial centerline velocity $U$

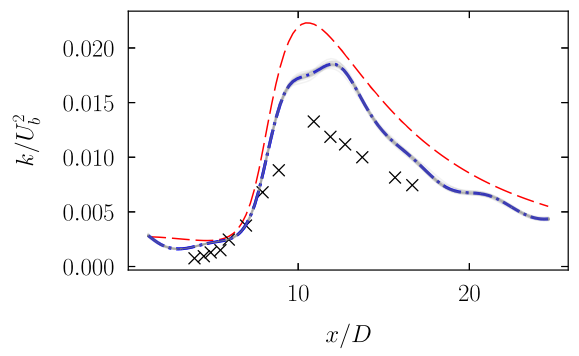

c) axial centerline TKE $\boldsymbol{k}$

Fig. 18 Inferred TKE and dissipation rate (the posterior sample mean) along the axial centerline for the realistic case 5.

nozzle exit velocity and nozzle diameter. We compare the inferred TKE with experiments as shown in Fig. 18c, since only turbulent kinetic energy is available from experimental measurements. The TKE is improved noticeably compared to the baseline but still exhibits discrepancies from the experimental measurements. That is likely due to the mixture of the RANS model uncertainty and acoustic model uncertainty, as in case 4 where the overestimation of the noise model parameter $A$ leads to underestimation of the TKE.

The errors between the experimental data and the assimilated results are summarized in Table $\underline{3}$. The centerline velocity and noise spectrum are in good agreement with the experimental data, reducing from $8.14 \%$ and $12.0 \%$ to the $2.35 \%$ and $0.036 \%$, respectively. On the other hand, the error of TKE is reduced from $73.9 \%$ to $48.3 \%$. Further improvement in the inference of the TKE requires additional observation or physical constraints, as in case 4.

\section{Discussion}

\section{A. Computational Cost}

We use 50 samples to compute the ensemble covariance and need 10 iterations to achieve statistical convergences in this work. Therefore, each inversion needs to perform the forward model 500 times. The CPU time of running the forward model is around $10 \%$ of the baseline case, since the turbulence model is not incorporated. We run the forward model simultaneously on 50 cores without communications between processes. Hence, the total wall time is not increased compared to the baseline case running on a single CPU core. The propagation step is responsible for the main computational cost, while the CPU time associated with the KL expansion and Kalman update can be neglected.

\section{B. IIl-Posedness of the Acoustic Inversion Problem}

The results presented in Sec. IV have shown that the framework can reconstruct the noise spectra and velocity in good agreement with the reference data. However, the inferred TKE $k$ and dissipation rate $\varepsilon$ still have noticeable discrepancies with the reference. That means that the acoustic inversion problem is ill-posed; that is, the solution of the inverse problem is not unique, and different turbulence fields can lead to similar noise spectra. To illustrate the ill-posedness of the acoustic inversion problem, we present the spatial contribution to the overall noise prediction. Specifically, we plot the noise spectral density contributed from each 5 diameter domain for case $2 a$ of assimilating the noise spectra data. The results are presented in Fig. 19. It clearly

Table 3 Results of the prediction error in the velocity field $U$, the noise spectra SPL, and the TKE $k$ for the realistic case

\begin{tabular}{lccc}
\hline \hline Case & err, $U$ & err, SPL & err, $k$ \\
\hline Baseline & $8.14 \%$ & $12.04 \%$ & $73.88 \%$ \\
5 (realistic case) & $2.35 \%$ & $0.036 \%$ & $48.30 \%$ \\
\hline \hline
\end{tabular}

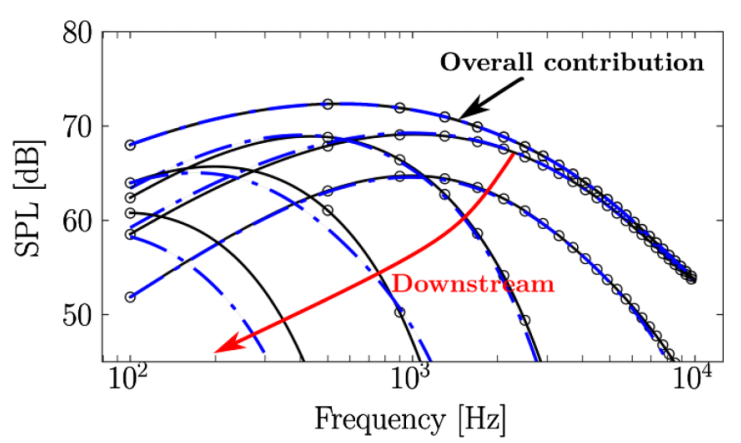

Fig. 19 Contribution of each 5 diameter slice to the noise spectral density. The blue/dashed line indicates the inferred results and the black/solid line indicates the reference results.

shows that the turbulence near the nozzle is responsible for the highfrequency noise, while the downstream mainly contributes to the low-frequency noise. It is reasonable since the small-scale eddies near the nozzle produce the high-frequency noise while the largescale eddies at the downstream mainly produce the noise with low frequency. However, the spectra from the first three slices have a very good agreement with the reference data in the high-frequency range but overestimate slightly in the low-frequency range, while the last two slices at the downstream underestimate the SPL spectrum. Thus, the discrepancy from the last two slices counteracts the first three slices, leading to the good agreement with reference data in the overall prediction of the SPL spectrum. This explains why the discrepancy in the inferred quantities has few effects on the noise prediction. Also, it illustrates the ill-posedness of the acoustic inversion problem, that is, different turbulent fields can produce the similar noise spectrum. Moreover, when considering uncertainties in both the noise model parameters and RANS-modeled quantities, the illposedness of the problem will further increase. As shown in case 4, the noise model parameters and the RANS-modeled quantities are difficult to infer simultaneously, even though the velocity and noise spectra are reconstructed well. That is likely due to the counteraction between the RANS model error and the acoustic model error. Specifically, the underestimation of the turbulent kinetic energy is compensated for by the noise model parameters as in case 4 . To alleviate this issue, additional regularization (e.g., smoothness) or observation data (e.g., noise spectrum observed at the downstream) need to be taken into account. Finally, when applicable, physical boundary conditions on the inferred field [56] and information on the partial differential equations describing these fields [57] can also help reducing the ill-posedness. These issues will be investigated in future research.

\section{Success and Limitations of Current Framework}

The present work demonstrates that the EnKF-based acoustic inversion framework is able to infer the TKE and dissipation rate 
based on the far-field noise measurements. The far-field noise spectrum and flowfields can be reconstructed effectively with the inferred quantities. It is shown that the best reconstruction results are obtained by assimilating the noise spectra and axial centerline velocity simultaneously. Moreover, the inferred model discrepancy in the RANS and noise model is promising to aid low-order jet noise modeling. Also, we show that the inferred noise source is possible to predict the far-field noise at unobserved locations. It would be of interest to predict the sound pressure level at unobserved locations based on the noise measurement from several microphones.

As for the limitations of this work, we mainly focus on using synthetic noise data at the observation angle of $90 \mathrm{deg}$ and assume a perfect noise model without errors. Under this assumption, the noise spectra at unobserved positions can be related to those at the observed locations based on the distance $r$ without using the acoustic inversion. Further validations need to be conducted to extend the framework for the noise prediction at the downstream but are out of the scope of the present work. To make the framework practical for realistic applications, both the RANS model and noise model uncertainties need to be considered and quantified, and other observations of far-field noise at the downstream are needed to alleviate the ill-posedness of the inverse problem. In spite of these limitations, the framework provides a data-driven route for inverse modeling of the jet noise sources and also shows the possibility to predict the noise spectrum at unobserved locations based on noise measurements at observed locations.

\section{Conclusions}

Rapid prediction of jet noise is critical for the optimal design of modern jet engines. However, the predominant low-order modeling of the jet noise source, for example, the RANS-based method, often leads to inaccurate predictions, particularly in the high-frequency range and geometrically complex jets with chevron or offset streams. Further improvements are demanding for the jet noise modeling. This work proposes an acoustic inversion method to reduce the uncertainties in the RANS-based jet noise prediction by incorporating the far-field noise measurements of subsonic jet flows. The ensemble Kalman filter is employed to assimilate observation data, including the centerline velocity and the acoustic noise data. We show that the noise data are very effective to reconstruct the turbulent kinetic energy of the jet flow, and the inferred noise source is able to improve the prediction in the mean velocity field and the noise at other locations. Besides, the proposed EnKF-based acoustic inversion method is feasible to assimilate disparate data (e.g., the velocity and the noise spectra) to enhance the reconstruction of the near-field turbulence and far-field noise spectra. Further investigations are conducted to take into account the uncertainties in the noise model parameters and demonstrate the practicality of the method in a realistic application with experimental measurements. The proposed EnKF-based acoustic inversion framework opens up the possibility of using the far-field noise measurements to reduce uncertainties in the RANS and noise model, which provides a novel route for data-augmented jet noise modeling.

\section{Appendix: Sensitivity Study of Hyper Parameters in Acoustic Inversion}

Here we present a sensitivity study of the variance $\sigma$ and the correlation length $l$ for the field of $k$ and $\varepsilon$ and the variance $\sigma_{A}, \sigma_{c_{l}}$, $\sigma_{c_{\tau}}$ for the parameters $A, c_{l}, c_{\tau}$ based on case 4 . The results are summarized in Table A1. It can be seen that the proposed method is robust for a large range of the parameters that lead to satisfactory reconstructions of the velocity, noise spectra, and the TKE and dissipation rate. However, the results are sensitive to small values in the variance $\sigma, \sigma_{A}, \sigma_{c_{l}}, \sigma_{c_{\tau}}$ for case 4 . That is likely due to the fact that the too-small variance leads to overconfidence in the initial value, and the reconstruction results can deviate much from the observation data.
Table A1 Summary of the parametric sensitivity study in case 4

\begin{tabular}{lcrlcc}
\hline \hline Parameter & Value & err, $U$ & err, SPL & err, $k$ & err, $\varepsilon$ \\
\hline$\sigma$ & 0.01 & $11.45 \%$ & $5.20 \%$ & $27.55 \%$ & $26.84 \%$ \\
& $\mathbf{0 . 1}$ & $1.21 \%$ & $0.010 \%$ & $14.03 \%$ & $26.02 \%$ \\
$l$ & 1 & $3.10 \%$ & $0.026 \%$ & $16.81 \%$ & $15.91 \%$ \\
& 0.01 & $3.48 \%$ & $0.19 \%$ & $19.35 \%$ & $26.35 \%$ \\
& $\mathbf{0 . 1}$ & $1.21 \%$ & $0.010 \%$ & $14.03 \%$ & $26.02 \%$ \\
$\sigma_{A}$ & 1 & $1.65 \%$ & $0.094 \%$ & $11.98 \%$ & $20.75 \%$ \\
& 1 & $2.69 \%$ & $0.040 \%$ & $17.20 \%$ & $30.04 \%$ \\
& $\mathbf{2}$ & $1.21 \%$ & $0.010 \%$ & $14.03 \%$ & $26.02 \%$ \\
$\sigma_{c_{l}}$ & 4 & $1.31 \%$ & $0.029 \%$ & $14.97 \%$ & $26.80 \%$ \\
& $0.05 \%$ & $13.80 \%$ & $4.85 \%$ & $50.78 \%$ & $38.85 \%$ \\
& $\mathbf{0 . 1}$ & $1.21 \%$ & $0.010 \%$ & $14.03 \%$ & $26.02 \%$ \\
$\sigma_{c_{\tau}}$ & 0.2 & $2.14 \%$ & $0.0091 \%$ & $15.03 \%$ & $24.99 \%$ \\
& $0.05 \%$ & $9.38 \%$ & $1.01 \%$ & $24.87 \%$ & $47.14 \%$ \\
& $\mathbf{0 . 1}$ & $1.21 \%$ & $0.010 \%$ & $14.03 \%$ & $26.02 \%$ \\
\hline \hline & 0.2 & $2.39 \%$ & $0.046 \%$ & $15.42 \%$ & $26.79 \%$ \\
\hline
\end{tabular}

The values in bold indicate the reference values.

\section{Acknowledgments}

XLZ, TW, and GH are supported by the National Science Foundation of China Basic Science Center Program for Multiscale Problems in Nonlinear Mechanics (no. 11988102), the Strategic Priority Research Program of the Chinese Academy of Sciences (XDB22040104), and the Key Research Program of Frontier Sciences of the Chinese Academy of Sciences (QYZDJ-SSWSYS002). HX did not receive external funding for this research.

\section{References}

[1] Lele, S. K., and Nichols, J. W., "A Second Golden Age of Aeroacoustics?" Philosophical Transactions of the Royal Society A, Vol. 372, No. 2022, 2014, Paper 20130321. https://doi.org/10.1098/rsta.2013.0321

[2] Bodony, D. J., and Lele, S. K., "Current Status of Jet Noise Predictions Using Large-Eddy Simulation," AIAA Journal, Vol. 46, No. 2, 2008, pp. 364-380. https://doi.org/10.2514/1.24475

[3] Brès, G. A., and Lele, S. K., "Modelling of Jet Noise: A Perspective from Large-Eddy Simulations," Philosophical Transactions of the Royal Society A, Vol. 377, No. 2159, 2019, Paper 20190081.

https://doi.org/10.1098/rsta.2019.0081

[4] Jordan, P., and Colonius, T., "Wave Packets and Turbulent Jet Noise," Annual Review of Fluid Mechanics, Vol. 45, Jan. 2013, pp. 173-195. https://doi.org/10.1146/annurev-fluid-011212-140756

[5] Schmidt, O. T., Towne, A., Rigas, G., Colonius, T., and Brès, G. A., "Spectral Analysis of Jet Turbulence," Journal of Fluid Mechanics, Vol. 855, Nov. 2018, pp. 953-982. https://doi.org/10.1017/jfm.2018.675

[6] Morris, P., and Boluriaan, S., "The Prediction of Jet Noise from CFD Data," AIAA Paper 2004-2977, 2004. https://doi.org/10.2514/6.2004-2977

[7] Engel, R. C., Silva, C. R., and Deschamps, C. J., "Application of RANSBased Method to Predict Acoustic Noise of Chevron Nozzles," Applied Acoustics, Vol. 79, May 2014, pp. 153-163. https://doi.org/10.1016/j.apacoust.2013.12.019

[8] Bridges, J. E., "Rapid Prediction of Installed Jet Noise from RANS," AIAA Paper 2020-2732, 2019. https://doi.org/10.2514/6.2019-2732

[9] Tam, C. K., Viswanathan, K., Ahuja, K., and Panda, J., "The Sources of Jet Noise: Experimental Evidence," Journal of Fluid Mechanics, Vol. 615, Nov. 2008, pp. 253-292. https://doi.org/10.1017/S0022112008003704

[10] Tam, C. K., "Jet Noise: Since 1952," Theoretical and Computational Fluid Dynamics, Vol. 10, Jan. 1998, pp. 393-405. https://doi.org/10.1007/s001620050072

[11] Tam, C. K., and Auriault, L., "Jet Mixing Noise from Fine-Scale Turbulence," AIAA Journal, Vol. 37, No. 2, 1999, pp. 145-153. https://doi.org/10.2514/2.691

[12] Morris, P. J., and Farassat, F., "Acoustic Analogy and Alternative Theories for Jet Noise Prediction," AIAA Journal, Vol. 40, No. 4, 
2002, pp. 671-680.

https://doi.org/10.2514/2.1699

[13] Leib, S. J., and Goldstein, M. E., "Hybrid Source Model for Predicting High-Speed Jet Noise," AIAA Journal, Vol. 49, No. 7, 2011, pp. 13241335.

https://doi.org/10.2514/1.J050707

[14] Ilário, C. R., Azarpeyvand, M., Rosa, V., Self, R. H., and Meneghini, J. R., "Prediction of Jet Mixing Noise with Lighthill's Acoustic Analogy and Geometrical Acoustics," Journal of the Acoustical Society of America, Vol. 141, No. 2, 2017, pp. 1203-1213. https://doi.org/10.1121/1.4976076

[15] Papamoschou, D., "Modelling of Noise Reduction in Complex Multistream Jets," Journal of Fluid Mechanics, Vol. 834, Jan. 2018, pp. 555599. https://doi.org/10.1017/jfm.2017.730

[16] Glegg, S., and Devenport, W., Aeroacoustics of Low Mach Number Flows: Fundamentals, Analysis, and Measurement, Academic Press, New York, 2017

[17] Casalino, D., Diozzi, F., Sannino, R., and Paonessa, A., "Aircraft Noise Reduction Technologies: A Bibliographic Review," Aerospace Science \& Technology, Vol. 12, No. 1, 2008, pp. 1-17. https://doi.org/10.1016/j.ast.2007.10.004

[18] Bridges, J., Khavaran, A., and Hunter, C., "Assessment of Current Jet Noise Prediction Capabilities," AIAA Paper 2008-2933, 2008. https://doi.org/10.2514/6.2008-2933

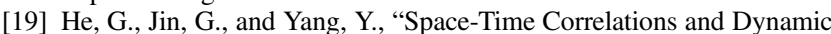
Coupling in Turbulent Flows," Annual Review of Fluid Mechanics, Vol. 49, Jan. 2017, pp. 51-70. https://doi.org/10.1146/annurev-fluid-010816-060309

[20] $\mathrm{Wu}, \mathrm{T}$. , and He, G., "Local Modulated Wave Model for the Reconstruction of Space-Time Energy Spectra in Turbulent Flows," Journal of Fluid Mechanics, Vol. 886, March 2020. https://doi.org/10.1017/jfm.2019.1044

[21] Franceschini, L., Sipp, D., and Marquet, O., "Mean-Flow Data Assimilation Based on Minimal Correction of Turbulence Models: Application to Turbulent High Reynolds Number Backward-Facing Step," Physical Review Fluids, Vol. 5, No. 9, 2020, Paper 094603. https://doi.org/10.1103/PhysRevFluids.5.094603

[22] Mons, V., Chassaing, J.-C., Gomez, T., and Sagaut, P., "Reconstruction of Unsteady Viscous Flows Using Data Assimilation Schemes," Journal of Computational Physics, Vol. 316, July 2016, pp. 255-280. https://doi.org/10.1016/j.jcp.2016.04.022

[23] He, C., Liu, Y., and Gan, L., "A Data Assimilation Model for Turbulent Flows Using Continuous Adjoint Formulation," Physics of Fluids, Vol. 30, No. 10, 2018, Paper 105108. https://doi.org/10.1063/1.5048727

[24] Labahn, J. W., Wu, H., Harris, S. R., Coriton, B., Frank, J. H., and Ihme, M., "Ensemble Kalman Filter for Assimilating Experimental Data into Large-Eddy Simulations of Turbulent Flows," Flow, Turbulence and Combustion, Vol. 105, Dec. 2019, pp. 1-33.

https://doi.org/10.1007/s10494-019-00093-1

[25] Pickering, E., Rigas, G., Schmidt, O. T., Sipp, D., and Colonius, T., "Optimal Eddy Viscosity for Resolvent-Based Models of Coherent Structures in Turbulent Jets," Journal of Fluid Mechanics, Vol. 917, June 2021. https://doi.org/10.1017/jfm.2021.232

[26] Wei, M., and Freund, J. B., "A Noise-Controlled Free Shear Flow," Journal of Fluid Mechanics, Vol. 546, Jan. 2006, p. 123. https://doi.org/10.1017/S0022112005007093

[27] Freund, J. B., "Adjoint-Based Optimization for Understanding and Suppressing Jet Noise," Journal of Sound and Vibration, Vol. 330, No. 17, 2011, pp. 4114-4122. https://doi.org/10.1016/i.jsv.2011.02.009

[28] Schulze, J., Schmid, P., and Sesterhenn, J., "Iterative Optimization Based on an Objective Functional in Frequency-Space with Application to Jet-Noise Cancellation," Journal of Computational Physics, Vol. 230, No. 15, 2011, pp. 6075-6098. https://doi.org/10.1016/j.jcp.2011.04.014

[29] Zhou, B. Y., Albring, T. A., Gauger, N. R., Economon, T. D., and Alonso, J. J., "An Efficient Unsteady Aerodynamic and Aeroacoustic Design Framework Using Discrete Adjoint," AIAA Paper 2016-3369, 2016. https://doi.org/10.2514/6.2016-3369

[30] Iglesias, M. A., Law, K. J., and Stuart, A. M., "Ensemble Kalman Methods for Inverse Problems," Inverse Problems, Vol. 29, No. 4, 2013, Paper 045001. https://doi.org/10.1088/0266-5611/29/4/045001

[31] Wang, J.-X., Tang, H., Xiao, H., and Weiss, R., "Inferring Tsunami Flow Depth and Flow Speed from Sediment Deposits Based on Ensemble
Kalman Filtering," Geophysical Journal International, Vol. 212, No. 1, 2018, pp. 646-658.

https://doi.org/10.1093/gji/ggx435

[32] Darakananda, D., da Silva, A. F. C., Colonius, T., and Eldredge, J. D., "Data-Assimilated Low-Order Vortex Modeling of Separated Flows," Physical Review Fluids, Vol. 3, No. 12, 2018, Paper 124701. https://doi.org/10.1103/PhysRevFluids.3.124701

[33] Kato, H., Yoshizawa, A., Ueno, G., and Obayashi, S., "A Data Assimilation Methodology for Reconstructing Turbulent Flows Around Aircraft," Journal of Computational Physics, Vol. 283, Feb. 2015, pp. 559581.

https://doi.org/10.1016/j.jcp.2014.12.013

[34] Zhang, X.-L., Michelén-Ströfer, C. A., and Xiao, H., "Regularized Ensemble Kalman Methods for Inverse Problems," Journal of Computational Physics, Vol. 416, Sept. 2020, Paper 109517. https://doi.org/10.1016/j.jcp.2020.109517

[35] Xiao, H., Wu, J.-L., Wang, J.-X., Sun, R., and Roy, C., "Quantifying and Reducing Model-Form Uncertainties in Reynolds-Averaged NavierStokes Simulations: A Data-Driven, Physics-Informed Bayesian Approach," Journal of Computational Physics, Vol. 324, Nov. 2016, pp. 115-136. https://doi.org/10.1016/j.jcp.2016.07.038

[36] Zhang, X.-L., Xiao, H., Gomez, T., and Coutier-Delgosha, O., "Evaluation of Ensemble Methods for Quantifying Uncertainties in SteadyState CFD Applications with Small Ensemble Sizes," Computers \& Fluids, Vol. 203, May 2020, Paper 104530.

https://doi.org/10.1016/j.compfluid.2020.104530

[37] Venkatesh, S. R., Polak, D. R., and Narayanan, S., "Beamforming Algorithm for Distributed Source Localization and its Application to Jet Noise," AIAA Journal, Vol. 41, No. 7, 2003, pp. 1238-1246. https://doi.org/10.2514/2.2092

[38] Papamoschou, D., and Dadvar, A., "Localization of Multiple Types of Jet Noise Sources," AIAA Paper 2006-2644, 2006. https://doi.org/10.2514/6.2006-2644

[39] Gerstoft, P., and Mecklenbräuker, C. F., "Ocean Acoustic Inversion with Estimation of a Posteriori Probability Distributions," Journal of the Acoustical Society of America, Vol. 104, No. 2, 1998, pp. 808-819. https://doi.org/10.1121/1.423355

[40] Siderius, M., Nielsen, P. L., and Gerstoft, P., "Range-Dependent Seabed Characterization by Inversion of Acoustic Data from a Towed Receiver Array," Journal of the Acoustical Society of America, Vol. 112, No. 4, 2002, pp. 1523-1535. https://doi.org/10.1121/1.1502264

[41] Wood, T. H., and Grace, S. M., "Inverse Aeroacoustic Problem for a Rectangular Wing," AIAA Journal, Vol. 38, No. 2, 2000, pp. 203-210. https://doi.org/10.2514/2.963

[42] Zhao, Q., and Li, X., "An Inverse Aeroacoustic Problem with Aerodynamic Constraint for a Helicopter Rotor," AIAA Paper 2018-3602, 2018. https://doi.org/10.2514/6.2018-3602

[43] Hursky, P., Porter, M. B., Cornuelle, B. D., Hodgkiss, W. S., and Kuperman, W. A., "Adjoint Modeling for Acoustic Inversion," Journal of the Acoustical Society of America, Vol. 115, No. 2, 2004, pp. 607-619. https://doi.org/10.1121/1.1636760

[44] Kim, D., Lee, K., and Seong, W., "Non-Cavitating Propeller Noise Modeling and Inversion," Journal of Sound and Vibration, Vol. 333, No. 24, 2014, pp. 6424-6437. https://doi.org/10.1016/j.jsv.2014.07.025

[45] Launder, B. E., and Sharma, B., "Application of the Energy-Dissipation Model of Turbulence to the Calculation of Flow near a Spinning Disc," Letters in Heat and Mass Transfer, Vol. 1, No. 2, 1974, pp. 131-137. https://doi.org/10.1016/0094-4548(74)90150-7

[46] Tam, C. K. W., and Auriault, L., "Mean Flow Refraction Effects on Sound Radiated from Localized Sources in a Jet," Journal of Fluid Mechanics, Vol. 370, Sept. 1998, pp. 149-174. https://doi.org/10.1017/S0022112098001852

[47] Le Matre, O., and Knio, O. M., Spectral Methods for Uncertainty Quantification: With Applications to Computational Fluid Dynamics, Springer Science \& Business Media, Berlin, 2010, pp. 18-22.

[48] Zhang, X.-L., Xiao, H., He, G., and Wang, S., "Assimilation of Disparate Data for Enhanced Reconstruction of Turbulent Mean Flows," Computers \& Fluids, Vol. 224, June 2021, Paper 104962. https://doi.org/10.1016/j.compfluid.2021.104962

[49] Michelén Ströfer, C. A., Zhang, X.-L., and Xiao, H., "DAFI: An OpenSource Framework for Ensemble-Based Data Assimilation and Field Inversion," Communications in Computational Physics, Vol. 29, No. 5, 2021, pp. 1583-1622. https://doi.org/10.4208/cicp.OA-2020-0178

[50] Lau, J. C., Morris, P. J., and Fisher, M. J., "Measurements in Subsonic and Supersonic Free Jets Using a Laser Velocimeter,' Journal of Fluid 
Mechanics, Vol. 93, No. 1, 1979, pp. 1-27.

https://doi.org/10.1017/S0022112079001750

[51] OpenCFD, OpenFOAM User Guide, 2018. https://www.openfoam. com/documentation/user-guide [retrieved 1 June 2020].

[52] Viswanathan, K., Shur, M., Spalart, P., and Strelets, M. K., "Comparisons Between Experiment and Large-Eddy Simulation for Jet Noise," AIAA Journal, Vol. 45, No. 8, 2007, pp. 1952-1966. https://doi.org/10.2514/1.25892

[53] Tahry, S. H. E., " $k-\epsilon$ Equation for Compressible Reciprocating Engine Flows," Journal of Energy, Vol. 7, No. 4, 1983, pp. 345-353. https://doi.org/10.2514/3.48086

[54] Rosa, V., Deschamps, C. J., Salazar, J. P. L. C., and Ilário, C. R. S., "Comparison of RANS-Based Jet Noise Models and Assessment of a Ray Tracing Method," Journal of the Brazilian Society of Mechanical Sciences and Engineering, Vol. 39, No. 6, 2017, pp. $1-14$.

https://doi.org/10.1007/s40430-017-0746-4
[55] Lau, J., "Effects of Exit Mach Number and Temperature on Mean-Flow and Turbulence Characteristics in Round Jets," Journal of Fluid Mechanics, Vol. 105, April 1981, pp. 193-218. https://doi.org/10.1017/S0022112081003170

[56] Michelén Ströfer, C. A., Zhang, X.-L., Xiao, H., and Coutier-Delgosha, O., "Enforcing Boundary Conditions on Physical Fields in Bayesian Inversion," Computer Methods in Applied Mechanics and Engineering, Vol. 367, Aug. 2020, Paper 113097. https://doi.org/10.1016/j.cma.2020.113097

[57] Wu, J.-L., Michelén Ströfer, C. A., and Xiao, H., "Physics-Informed Covariance Kernel for Model-Form Uncertainty Quantification with Application to Turbulent Flows," Computers \& Fluids, Vol. 193, Oct. 2019, Paper 104292.

https://doi.org/10.1016/j.compfluid.2019.104292

S. Fu 